\title{
Anatomically Informed Basis Functions for EEG Source Localization: Combining Functional and Anatomical Constraints
}

\author{
Christophe Phillips, ${ }^{*} \uparrow \uparrow$ Michael D. Rugg, ${ }^{*}$ and Karl J. Friston $\uparrow$
}

\author{
* Institute of Cognitive Neuroscience and $\uparrow$ Wellcome Department of Cognitive Neurology, Institute of \\ Neurology, University College London, London, United Kingdom; and $\uparrow$ Centre de Recherches du Cyclotron, \\ Université de Liège, 4000 Liège, Belgium
}

\begin{abstract}
Distributed linear solutions have frequently been used to solve the source localization problem in EEG. Here we introduce an approach based on the weighted minimum norm (WMN) method that imposes constraints using anatomical and physiological information derived from other imaging modalities. The anatomical constraints are used to reduce the solution space a priori by modeling the spatial source distribution with a set of basis functions. These spatial basis functions are chosen in a principled way using information theory. The reduced problem is then solved with a classical WMN method. Further (functional) constraints can be introduced in the weighting of the solution using fMRI brain responses to augment spatial priors. We used simulated data to explore the behavior of the approach over a range of the model's hyperparameters. To assess the construct validity of our method we compared it with two established approaches to the source localization problem, a simple weighted minimum norm and a maximum smoothness (Loreta-like) solution. This involved simulations, using single and multiple sources that were analyzed under different levels of confidence in the priors.
\end{abstract}

Key Words: EEG; source localization; distributed linear solution; informed basis functions; anatomical constraints; functional constraints.

\section{INTRODUCTION}

The aim of functional neuroimaging is to understand the functional organization of the brain. This aim incorporates several aspects of functional neuroanatomy: the location of processing areas, the time course or dynamics of their activities, and the nature of their interactions. Changes in neuronal activity induce variations in cerebral metabolism, blood flow, blood volume, and blood oxygenation (Frackowiak et al, 1997) and electromagnetic fields (Nunez, 1981; Hämäläinen et al., 1993; Malmivuo and Plonsey, 1995). Changes in these hemodynamic and electromagnetic signals can be measured by several noninvasive techniques, such as positron emission tomography (PET), functional magnetic resonance imaging (fMRI), electroencephalography (EEG), and magnetoencephalography (MEG).

Hemodynamic responses in the brain are recorded by PET and fMRI with a good spatial resolution, on the order of millimeters. These indices of neuronal activity are temporally limited by the latency and slow time constants of the hemodynamic response. In contrast direct measurements of the electromagnetic fields (EEG and MEG) produced by neuronal activity have a temporal resolution of less than $1 \mathrm{~ms}$.

However, the problem of recovering volume current sources from superficial electromagnetic measurement is intrinsicly ill-posed (von Helmholtz, 1853) and the spatial configuration of neuronal activity cannot be determined uniquely, based on EEG and/or MEG recordings alone (Nunez, 1981). To remove the nonuniqueness of this so-called inverse problem, assumptions about the solution must be made, so as to obtain a unique and "optimal" solution according to the criteria employed.

One common approach is to assume that the EEG/ MEG signals are generated by a relatively small number of focal sources (Miltner et al., 1994; Scherg and Ebersole, 1994; Scherg et al, 1999; Aine et al, 2000), each of which can be modeled as a single fixed or reorientating dipole. The idea is to render the inverse problem overdetermined by considering fewer unknown parameters than the independent measurements available. The locations, orientations, and strengths of these "equivalent current dipoles" (ECD) can be estimated by minimizing the difference between the predicted and the actual EEG/MEG measurements. An additional constraint can be derived from the assumption that the sources are temporally independent (Mosher et al, 1992). The fitting procedure involves a multidimensional, nonlinear optimization procedure. As the time required to solve the optimization problem grows geometrically with the number of ECDs, the global optimum can be found 
only for models involving very few ECDs. For models with a large number of ECDs, approximate techniques have to be used, where the solution depends upon the initial estimate of the locations and orientations of the dipoles. In all ECD-based methods, the solution depends heavily on the number of dipoles assumed but, in general, the actual number of ECD cannot be determined a priori.

Another approach is to consider a priori all possible fixed source locations. The problem of determining the strength of each dipole (or the two dipole components: orientation and strength) then becomes a linear one. This continuous current source model is more biologically plausible (for sufficiently dense source distributions) than the ECD model. Given the ill-posed nature of the inverse problem constraints are required to ensure the likelihood of the data or the log posterior of the conditional estimators has a unique maximum. The exact nature of these constraints operationally defines what is meant by a "good" solution. A good solution is one that jointly maximizes the likelihood of the data while minimizing a cost function of the constraints. This cost function can be construed as embodying prior information such that the "best" estimate maximizes the log posterior (i.e., the most likely estimate given the data). When the constraints have been chosen, a unique solution obtains through minimizing the deviation from these constraints (within the solution space that minimizes the residuals of the fit). For example, existing methods use one of the following constraints: probabilistic (Greenblatt, 1993; Baillet and Garnero, 1997), maximum entropy (Huang et al, 1997), minimum L1-norm (Uutela et al., 1999), (weighted) minimum L2-norm (WMN) (Sarvas, 1987; Hämäläinen and Ilmoniemi, 1994; Brooks et al., 1999), maximum smoothness (MS) ("low-resolution electromagnetic tomography" or "Loreta") (Pascual-Marqui et al., 1994; Pascual-Marqui, 1995, 1999), or optimal resolution (Backus and Gilbert, 1970; Grave de Peralta Menendez et al., 1997; Grave de Peralta Menendez and Gonzalez Andino, 1999). Although they are mathematically tractable, not all the above constraints are based on actual brain physiology and anatomy and they can lead to relatively poor spatial resolution or biased solutions.

The weighted minimum L2-norm constraint has two great advantages: the method provides a linear analytical solution that can be easily and directly computed, and different kinds of priors (whether framed explicitly in a Bayesian sense or not) can be employed. Here we introduce an approach based on the WMN method that imposes constraints using anatomical and physiological information derived from other imaging modalities. Three assumptions, based on neuroanatomy and spatial coherence, can be made about the sources of brain electromagnetic activity (Nunez, 1981; Hämäläinen et al., 1993; Dale and Sereno, 1993): they are located in gray matter, they are oriented orthogonally to the cortical sheet, and, for a sufficiently dense dipole distribution, they possess locally coherent activity (or smooth activity along the cortical sheet). In our approach these constraints are used to reduce the solution space a priori by modeling the spatial source distribution with a set of basis functions. Such an approach has already been applied in the analysis of fMRI activation studies (Kiebel et al, 2000). These spatial basis functions are chosen in a principled way using information theory. The reduced problem is then solved with a classical WMN method. Further constraints can be introduced in the weighting of the WMN solution using information derived from hemodynamic measures of brain activity as spatial priors (Rugg, 1998; Liu et al, 1998; Dale et al, 2000). These constraints are predicated on the conjecture that the synaptic currents generating EEG/MEG signals also impose metabolic demands, which lead to a hemodynamic response measurable by PET or fMRI. It is possible that the coupling between electromagnetic (EEG/ MEG) and hemodynamic (PET/fMRI) signals is not necessarily well behaved or deterministic (Rugg, 1999). Therefore, the prior location information derived from hemodynamic measurements should be regarded as probabilistic.

This paper is divided into three sections. In the first section, the theoretical background and operational details of our approach are described. In the second section, the method is demonstrated using a realistic head model based on a structural MRI. In the third section, we used simulated data to explore the behavior of the approach over a range of values of model hyper-parameters, e.g. different degrees of spatial coherence. To assess the construct validity of our method we compare it with two established approaches to the source localization problem, simple weighted minimum norm and a maximum smoothness (Loreta-like) solution. This involved an extensive set of simulations using single and multiple source sets analyzed using, for all methods, different levels of confidence in certain priors. This paper focuses on the theory and limiting behavior in the absence of noise. A companion paper (C. Phillips et al, unpublished) addresses the issues engendered by measurement noise.

In the rest of this paper, $\mathrm{a}, \alpha$, a, and A will, respectively, represent a scalar, a vector of size $3 \times 1$, a vector of any size $N_{a}$ x 1, and a matrix; $\mathbf{A}^{\mathbf{t}}$ will designate the transpose of $\mathbf{A}$.

\section{METHODS}

Theory

The General Approach

In the absence of any a priori information, the sources of the EEG signal can be modeled by a fixed, uniform, 
three-dimensional distribution of current dipoles throughout the entire brain volume. Each current dipole represents the coherent electrical activity of the brain over a small cubic volume. We make three strong assumptions, based on neuroanatomy and neurophysiology, about these dipoles: they are located in the the gray matter, their orientation is perpendicular to the cortical sheet, and they are spatially coherent. These constraints are implemented operationally using information derived from the subject's structural MRI (see Extracting the Constraints).

By fixing the orientation of each dipole, the source localization problem is reduced from a vectorial problem, where both the orientation and the amplitude of the dipoles are unknown, to a scalar problem, where only the amplitudes of the dipoles are unknown. For instantaneous data, the distributed source localization problem can be stated as

$$
\mathbf{v}+\varepsilon=\mathbf{L} \mathbf{j}
$$

where $\mathrm{v}$ (the electric potential at the electrodes) is a vector of size $N_{e}$ x $1, \varepsilon$ (the additive noise component) is a vector of size $N_{e}$ x 1, j (the (unknown) amplitude of each current dipole) is a vector of size $N_{i}$, x 1 , L (the lead field linking the current sources, $\mathbf{j}$, to the electrical potential, v) is a matrix of size $N_{e} \mathrm{x} N_{i}$, and the orientation of the current dipoles is embodied in the lead field matrix L, so that each element of the vector j represents only the amplitude of each dipole.

Having fixed the orientation of the dipoles, the two other anatomical assumptions described above, spatial smoothness and location within the gray matter, are used to establish a spatial basis set that models the source distribution. The two anatomical priors enter as constraints on the covariance structure of the source distribution and motivate the selection of the basis set. This set is calculated in a way that maximizes the mutual information between the original (and full) source distribution space and the reduced solution space spanned by the basis set (see Basis Function set).

If other prior knowledge about the location of electrical activity is available (e.g., from a functional MRI activation study), it may also help to constrain the source localization problem. This information can be regarded as a soft or probabilistic constraint (compared to the anatomical priors used to determine the spatial basis function set) and therefore enters during the second step of the method, i.e. when the solution (constrained by the basis functions) is identified by a weighted minimum L2-norm approach (see Minimum Norm Solution.

\section{Extracting the Constraints}

Gray matter density. First we segment the MR brain image into its principal partitions: gray matter, white matter, and cerebrospinal fluid (Ashburner and Friston, 1997). A gray matter density coefficient is then determined from the smoothed gray matter image at each dipole location. The coefficient varies in value from 0 , zero probability that the small cubic volume (i.e., voxel) surrounding the dipole is in gray matter, to 1 , certainty that the dipole is embedded in gray matter. These coefficients constitute the leading diagonal of matrix G of size $N_{j}$ x $N_{j}$.

Dipole orientation. Here the orientation of the dipoles are fixed perpendicular to the interface between gray and white matter pointing toward the outside of the brain. This orientation is obtained from the three-dimensional gradient of the smoothed white matter volume: The gradient vector field is oriented, at each location, in the direction of the largest variation of smoothed white matter density. The smoothing is anisotropic using a diffusion process (Perona and Malik, 1990),

$$
\frac{\delta u(\vec{r}, t)}{\delta t}=\nabla(c(\vec{r}) \nabla u(\vec{r}, t))
$$

where $u(\vec{r}, t)$ is the white matter density at location $\vec{r}$

for the virtual time instant $t$ of the diffusion process (at time $t=0, u(\vec{T}, 0)$ is the original unsmoothed white matter volume), $c(\vec{I})$ (the anisotropic diffusion coefficient) is set to a value corresponding to the gray matter density at location $\vec{r}$ (which remains constant throughout the diffusion process), and the degree of smoothness is determined by the duration $t$ of the diffusion process. The definition of $u(\vec{r}, t)$ and $c(\vec{r})$ ensures that white matter image $u(\vec{r})$ is smoothed in the direction of the highest gray matter density. The gradient $\nabla u_{\text {as }}(\vec{r})$ of the anisotropically smoothed white matter image $u_{a s}(\vec{r})$ is thus oriented perpendicular to the gray and white matter interface. This ensures that the gradient of the smoothed white matter image is appropriate for fixing the orientation of each dipole (cf. the approach of George et al. (1995)).

Spatial coherence. We then model the spatial coherence of the dipoles on the basis of their "connectivity." The $N_{j}$ x $N_{j}$ matrix D represents this spatial coherence. As the dipoles are spread on a regular three-dimensional grid, each dipole has at most 26 nearest neighbors. Not all neighbor dipoles are necessarily connected to the central 
one. Connectivity depends on the brain anatomy; e.g., dipoles located on opposite sides of a sulcus should not be connected.

The connectivity of a (central) dipole located at $\vec{r}$ with its nearest neighbors located at $\vec{r}+\vec{d} r$ is assessed using the orientation of the cortical surface. This orientation is determined by a first order approximation to the gradient $\nabla u_{a s}(\vec{r})$ of the anisotropically smoothed white matter $\left(u_{a s}(\vec{r})\right)$ around the central dipole:

$\nabla u_{a s}(\vec{r}+\overrightarrow{d r})=\nabla u_{a s}(\vec{r})+\overrightarrow{d r} \nabla^{2} u_{a s}(\vec{r})$.

This estimated orientation is compared to the empirical white matter gradient at the neighboring dipole locations. If the discrepancy is too large, the dipoles are considered disconnected, as for the case of dipoles on opposite sides of a sulcus. Otherwise the dipoles are considered connected. This gives a sparse connectivity matrix $\Delta$ with element 1 for a connection or 0 otherwise. The spatial coherence can be derived from $\Delta$ in a variety of ways. For example if we assume a simple spatial regression model for the spread of activity from one dipole to another, we obtain

$\mathbf{D}^{t} \mathbf{D}=\left(\mathbf{I}_{N_{j}}-\sigma \boldsymbol{\Delta}\right)^{-1}\left(\mathbf{I}_{N_{j}}-\sigma \boldsymbol{\Delta}\right)^{-t}$.

Alternatively we can assume some decreasing function of path length (e.g., Gaussian), where path length is the shortest path from one dipole to another based on $\Delta$, to generate $\mathrm{D}$.

\section{Basis Function Set}

With the matrices $\mathbf{G}$, gray matter density at the dipole locations, and $\mathbf{D}$, spatial coherence among the dipoles, a set of spatial basis functions that will allow the source distribution to be modeled with a substantial reduction in the uncertainty of the solution can be generated. From The General Approach, the noise-free problem is

$$
\mathbf{v}=\mathbf{L} \mathbf{j} \text {, }
$$

where $\mathrm{j}$ is unknown. To reduce the size of the solution space from $N_{j}$ to $N_{k}$, where $N_{k} \ll N_{j}, \mathbf{j}$ is modeled as $\tilde{\mathbf{j}}$,

$\mathbf{B}^{t} \mathbf{j}=\mathbf{k} \Rightarrow \tilde{\mathbf{j}}=\mathbf{B k}$,

where B is a $N_{j} \mathrm{x} N_{k}$ matrix that maximizes the mutual information (Jones, 1979) between $\mathbf{j}$ and $\mathbf{k}$,

$\mathbf{I}(\mathbf{j}, \mathbf{k})=\mathbf{H}(\mathbf{j})+\mathbf{H}(\mathbf{k})-\mathbf{H}(\mathbf{j} \cap \mathbf{k})=\mathbf{H}(\mathbf{k})$

because $\mathbf{H}(\mathbf{j})=\mathbf{H}(\mathbf{j} \cap \mathbf{k})$ where $\mathbf{H}(\mathbf{j})$ represents the entropy of $\mathrm{j}$. If $\mathrm{k}$ is multinomial, then

$\mathbf{H}(\mathbf{k}) \propto \ln \left(\left|\mathbf{C}_{\mathbf{k}}\right|\right)=\sum_{i} \ln \left(\lambda_{i}\right)$,

where $\mathbf{C}_{\mathbf{k}}=\left(\mathbf{k k}^{\mathbf{t}}\right)=\left(\mathbf{B}^{\mathbf{t}} \mathbf{j j}{ }^{\mathbf{t}} \mathbf{B}\right)=\mathbf{B}^{\mathbf{t}} \mathbf{C}_{\mathbf{j}} \mathbf{B}$ is the variance-covariance matrix of $\mathrm{k}$ and the $\lambda_{i}$ are $\mathbf{C}_{\mathbf{k}}{ }^{\prime}$ s eigenvalues. The expression to maximize is thus

$\ln \left|\mathbf{B}^{t} \mathbf{C}_{\mathbf{j}} \mathbf{B}\right|=\sum_{i} \ln \left(\lambda_{j}\right)$

Therefore the matrix $\mathbf{B}$ should comprise the eigenvectors of the variance- covariance matrix $C_{i}=\left(\mathbf{j j}^{\mathbf{t}}\right)$ corresponding to the highest eigenvalues $\lambda_{i}$.

Prior knowledge about $\mathbf{j}$, embodied in $\mathbf{D}$ and $\mathbf{G}_{1}$ can be included by noting

$$
\mathbf{C}_{\mathbf{j}}=\mathbf{G}^{t / 2} \mathbf{D}^{t} \mathbf{D G}^{1 / 2} \text {. }
$$

$\mathrm{B}$ is obtained from the eigenvector solution of $\mathrm{G}^{t / 2} \mathrm{D}^{t} \mathrm{DG}^{1 / 2}$ or equivalently by using the singular value decomposition of $\mathrm{DG}^{1 / 2}$ :

$$
\mathbf{U S W} \mathbf{W}^{t}=\operatorname{svd}\left(\mathbf{D G}{ }^{1 / 2}\right) \text {. }
$$

Columns of $\mathbf{W}$ corresponding to the highest singular values $\mathbf{S}$ are used to define $\mathbf{B}$ (see Step 1 : Estimation of Spatial Basis Functions for the threshold used).

In summary, the distributed source problem is reduced to finding the coefficients (k) of a set of spatial modes (B) or basis functions where, critically, these modes are chosen to preserve as much information about the distributed profile as possible. These modes are simply the eigenmodes or principle components of the source covariance $\left(\mathbf{C}_{\mathbf{j}}\right)$ predicted by our assumptions about the sources. 


\section{Minimum Norm Solution}

Given the spatial basis function set $\mathbf{B}$, the instantaneous source localization problem (1) can be reformulated as

$\mathbf{v}+\varepsilon=\mathbf{L B k}=\mathbf{L}_{\mathbf{B}} \mathbf{k}$.

To solve this reduced problem, a simple pseudo-inverse, e.g., Moore-Penrose pseudo-inverse, could be applied to $\mathbf{L}_{\mathbf{B}}$. However because of the ill-posed nature of the source localization problem, this unconstrained solution is generally inadequate. A regularization constraint on the solution can be applied to minimize some weighted norm $\|\mathbf{H} \mathbf{j}\|$ of the current density $\tilde{\mathbf{j}}=\mathbf{B k}$ or some weighted norm $\left\|\mathbf{H}_{\mathbf{B}} \mathbf{k}\right\|$ o $\mathrm{f}$ the basis coefficients $\mathrm{k}$.

$\mathbf{H}$ and $\mathbf{H}_{\mathbf{B}}$ specify the nature of the regularization required. Assuming that the noise $\varepsilon$ is characterized by the covariance matrix $\operatorname{cov}(\varepsilon)=\mathbf{C}_{\varepsilon}$, the weighted minimum norm problem or "Tikhonov regularization" (Tik-honov and Arsenin, 1977) is expressed as

$$
\hat{\jmath}=\arg \min _{\mathbf{j}}\left\{\left\|\mathbf{C}_{\varepsilon}^{-1 / 2}\left(\mathbf{L} \mathbf{j}-\mathbf{v}_{\varepsilon}\right)\right\|^{2}+\lambda^{2}\|\mathbf{H} \mathbf{j}\|^{2}\right\}
$$

and, incorporating the basis function coefficients $\mathbf{k}$,

$\hat{\mathbf{k}}=\arg \min _{\mathbf{k}}\left\{\left\|\mathbf{C}_{\varepsilon}^{-1 / 2}\left(\mathbf{L}_{\mathbf{B}} \mathbf{k}-\mathbf{v}_{\varepsilon}\right)\right\|^{2}+\lambda^{2}\left\|\mathbf{H}_{\mathbf{B}} \mathbf{k}\right\|^{2}\right\}$,

where $\mathbf{v}_{\varepsilon}=\mathbf{v}+\boldsymbol{\varepsilon}$ represents underlying signal with noise. Here $\lambda$ is a hyperparameter that controls the influence of the constraints relative to minimizing the error of the fit.

The solution of (14) should provide the best fit to the data while minimizing the constraint $\left\|\mathbf{H}_{\mathbf{B}} \mathbf{k}\right\|^{2}$. Such a solution has the form

$\hat{\mathbf{k}}=\mathrm{T}_{\mathrm{B}} \mathbf{v}_{\varepsilon} \Rightarrow \hat{\mathbf{j}}=\mathrm{BT}_{\mathrm{B}} \mathbf{v}_{\varepsilon}=\mathrm{Tv}_{\boldsymbol{\varepsilon}}$

where

$$
\begin{aligned}
\mathbf{T}_{\mathbf{B}} & =\left[\mathbf{L}_{\mathbf{B}}^{t} \mathbf{C}_{\varepsilon}^{-1} \mathbf{L}_{\mathbf{B}}+\lambda^{2}\left(\mathbf{H}_{\mathbf{B}}^{t} \mathbf{H}_{\mathbf{B}}\right)\right]^{-1} \mathbf{L}_{\mathbf{B}}^{t} \mathbf{C}_{\varepsilon}^{-1} \\
& =\left(\mathbf{H}_{\mathbf{B}}^{t} \mathbf{H}_{\mathbf{B}}\right)^{-1} \mathbf{L}_{\mathbf{B}}^{t}\left[\mathbf{L}_{\mathbf{B}}\left(\mathbf{H}_{\mathbf{B}}^{t} \mathbf{H}_{\mathbf{B}}\right)^{-1} \mathbf{L}_{\mathbf{B}}^{t}+\lambda^{2} \mathbf{C}_{\varepsilon}\right]^{-1}
\end{aligned}
$$

from the matrix inversion Lemma.

There is an important and useful connection with Bayesian estimates of the sources here, where under Gaussian assumptions, the conditional expectation or posterior mean of the sources $\mathbf{k}$ is given by

$$
\begin{aligned}
E\left(\mathbf{k} \mid \mathbf{v}_{\varepsilon}\right) & =\left[\mathbf{L}_{\mathbf{B}}^{t} \mathbf{C}_{\varepsilon}^{-1} \mathbf{L}_{\mathbf{B}}+\mathbf{C}_{\mathbf{k}}^{-1}\right]^{-1} \mathbf{L}_{\mathbf{B}}^{t} \mathbf{C}_{\varepsilon}^{-1} \mathbf{v}_{\varepsilon} \\
& =\mathbf{C}_{\mathbf{k}} \mathbf{L}_{\mathbf{B}}^{t}\left[\mathbf{L}_{\mathbf{B}} \mathbf{C}_{\mathbf{k}} \mathbf{L}_{\mathbf{B}}^{t}+\mathbf{C}_{\varepsilon}\right]^{-1} \mathbf{v}_{\varepsilon}
\end{aligned}
$$

where $\mathbf{C}_{\mathbf{k}}$ is the prior covariance of the sources. Comparing Eq. (17) with Eq. (16) provides the motivation for choosing forms of $\mathbf{H}_{\mathbf{B}}$ such that $\mathbf{H}_{\mathbf{B}} \propto \mathbf{C}_{\mathbf{k}}^{-1 / 2}$. We shall use this result later.

In the particular and theoretical case where the measurements are noise free, i.e., $\mathbf{v}_{\varepsilon}=\mathbf{v}$, the solution of Eq. (14) is obtained from Eq. (16) by taking the limit $\lambda \rightarrow 0$ (Rao and Mitra, 1973), i.e., minimizing the constraint $\left\|\mathbf{H}_{\mathbf{B}} \mathbf{k}\right\|^{2}$ after fitting the model perfectly:

$$
\mathbf{T}_{\mathrm{B}}=\left(\mathbf{H}_{\mathrm{B}}^{t} \mathbf{H}_{\mathrm{B}}\right)^{-1} \mathbf{L}_{\mathrm{B}}^{t}\left[\mathbf{L}_{\mathbf{B}}\left(\mathbf{H}_{\mathrm{B}}^{t} \mathbf{H}_{\mathbf{B}}\right)^{-1} \mathbf{L}_{\mathbf{B}}^{t}\right]^{-1}
$$

In the present paper, we use only noise-free simulated data; therefore the solutions presented are based on Eq. (18). Noise dependency will be addressed in a subsequent paper (C. Phillips et al., unpublished), where we will deal with the estimation of $\lambda$, which itself is quite a complicated issue. 


\section{Application of the Theory}

\section{Head and Source Model}

The method described above was applied to simulated data using a realistic head model generated from a Tlweighted structural MR image, the template MRI of the SPM99 software package (Wellcome Department of Cognitive Neurology, 1999). The lead field matrix L, the solution of the so-called "forward problem," was calculated with the "boundary element method" (BEM) (Hämäläinen and Sarvas, 1989; Ferguson and Stroink, 1997) for a set of 61 approximately equidistant electrodes and 12,300 dipoles within the brain volume arranged on a regular three-dimensional grid (interdi-pole distance $4 \mathrm{~mm}$ ). In the BEM, the head is modeled as three concentric volumes of isotropic conductivity: the brain, the skull, and the scalp volume. The dipoles are distributed within the brain volume.

\section{Step 1: Estimation of Spatial Basis Functions}

The MRI was segmented with the segmentation function of the SPM99 software package (Wellcome Department of Cognitive Neurology, 1999), and the gray matter image was averaged over each dipole cubic volume to provide the gray matter coefficient for the matrix G. The 12,300 dipoles noted under Head and Source Model are the dipoles, from the original full grid, located in voxels with gray matter density greater than 0.4 ; i.e., $40 \%$ (or more) of the voxel was gray matter or the probability of the voxel being gray matter was 0.4 (or more). This density value ensured that each dipole represents the electrical activity of a small volume of the brain containing a sufficient amount of gray matter. Selecting this subset of all potential dipoles represents a compromise between considering all the dipoles and considering those that potentially contribute a substantial signal (i.e., it is pointless to retain dipoles located in white matter or cerebrospinal fluid).

The numerical approximation of Eq. (2) was used to smooth the white matter;

$$
\begin{aligned}
u_{(i, j, k)}^{t+1}=u_{(i, j, k)}^{t}+ & \lambda\left[c_{N} \delta_{N} u+c_{S} \delta_{S} u+c_{E} \delta_{E} u\right. \\
& \left.+c_{W} \delta_{W} u+c_{U} \delta_{U} u+c_{A} \delta_{A} u\right]_{(i, j, k)}^{t},
\end{aligned}
$$

where $c_{(i, j, k)}$ is the gray matter density at voxel $(i, j, k), u_{(i, j, k)}^{t}$ is the white matter density at voxel $(i, j, k)$ at iteration $t$, and

$$
\begin{aligned}
& \delta_{N} u=u_{(i+1, j, k)}-u_{(i, j, k)} \quad c_{N}=\frac{c_{(i+1, j, k)}+c_{(i, j, k)}}{2} \\
& \delta_{S} u=u_{(i-1, j, k)}-u_{(i, j, k)} \quad c_{S}=\frac{c_{(i-1, j, k)}+c_{(i, j, k)}}{2}
\end{aligned}
$$

$\delta_{E} u, \delta_{W} u, c_{E}$, and $C_{W}$ are defined like $\delta_{N} u, v_{S} u, c_{N}$, and $c_{S}$ with a variation of the index $j$ and like $\delta_{U} u, \delta_{A} u, c_{U}$, and $c_{A}$ with a variation of the index $k$.

To ensure that the smoothing process is stable, it is necessary to select $\lambda$ such that $0<\lambda<1 / 8$. An isotropic diffusion process, i.e., with $c_{(i, j, k)}=1$ everywhere, approximates a Gaussian function of kernel $\sigma=\sqrt{2 \lambda t}$. By chosing $\sigma$ to be equal to the interdipole distance, the number $t$ of iterations is specified to give the required smoothing. Starting with $u^{0}$, the segmented white matter volume, Eq. (19) was applied iteratively until the desired smoothing was achieved. The gradient of the smoothed white matter was then calculated and sampled at the location of the dipoles. The successive steps of the MRI processing are summarized in Fig. 1.

Equation (3) was used to determine the connectivity of neighboring dipoles. The estimated orientation of neighboring dipoles was compared with their real orientation and if the angle between the orientations was greater than $\boldsymbol{\pi} / \mathbf{2}$, the dipoles were considered disconnected. Instead of using Eq. (4), we used a Gaussian function of path length. This allowed us to parameterize the spatial coherence in a way more useful to our purposes. The path length was calculated as follows: For each pair of connected dipoles, a parabolic curve joining the dipoles and fitting their orientation was calculated. The length of this curve was used as "path length." The distance between two dipoles that were not directly connected was obtained by looking for the shortest path (within an upper limit of $30 \mathrm{~mm}$ ) composed of connected dipoles.

Two degrees of spatial coherence were studied by using two different Gaussians, $\sigma=10 \mathrm{~mm}$ and $\sigma=5 \mathrm{~mm}$. For each of the two $\mathrm{D}$ matrices obtained, the singular vectors of $\mathrm{G}^{\mathrm{t} / 2} \mathrm{D}^{\mathrm{t}} \mathrm{DG} \mathrm{G}^{1 / 2}$ with normalized eigenvalues greater 
than unity were retained to form the basis set B. The ensuing spectrum of singular values is shown in Fig. 2.

The original solution space $\mathbf{j}$ of 12,300 dimensions was reduced to a space $\mathbf{k}$ of 621 and 1903 dimensions for the large $(\sigma=10 \mathrm{~mm})$ and small $(\sigma=5 \mathrm{~mm})$ coherences, respectively (a reduction of about 95 and $85 \%)$. The dimension of the reduced space corresponds to the number of orthonormal basis functions in $\mathbf{B}$. The solutions based on $\mathbf{T}_{\mathbf{B}}$ obtained using Eq. (18) will be referred to as the "Informed Basis Function" (IBF) solutions of kernel $5 \mathrm{~mm}$ (IBF5) or $10 \mathrm{~mm}$ (IBF10).

\section{Step 2: Minimum Norm Solution}

The (weighted) minimum norm solution depends on the specification of the constraints $\mathbf{H}_{\mathbf{B}}$ that enter into Eq. (14). As noted under Minimum Norm Solution, $\mathbf{H}_{\mathbf{B}}^{-} \propto \mathbf{C}_{\mathbf{k}}^{-1 / 2}$. Because the solution space has been reduced using the eigenvectors of $\mathbf{C}_{i}$, the source prior covariance matrix $\mathbf{C}_{k}$ could simply be its eigenvalues $\mathbf{C}_{k}^{-1 / 2}=\mathbf{S}^{-1}$, where $\mathbf{S}$ is the leading diagonal matrix of singular values from Eq. (11). This simple form for the constraints could be used directly.

However, further "soft" priors on the covariance of the sources can be included by specifying linearly separable components of the prior source covariance matrix, in addition to the "hard" constraints used to determine the spatial basis set. Each of these covariance components is controlled by its own hyperpa-rameter. There are two additional constraints considered here.

First, because superficial sources produce a stronger (and more focal) scalp electrical potential than deeper sources, shallow sources are "penalized" to ensure that sources are more likely to influence the electrical potential equally at the electrodes irrespective of depth (Pascual-Marqui et al., 1994; Grave de Peralta Menendez and Gonzalez Andino, 1998; Pascual-Marqui, 1999). This can be achieved by assuming that deeper sources have a larger variance than superficial sources. The depth is indexed by the norm of the source's lead field. Let the covariance component of this constraint be the diagonal matrix $\operatorname{diag}\left(\mathrm{L}^{\mathrm{t}} \mathrm{L}\right)^{-1}$. Using this diagonal matrix is equivalent to normalizing the column of the lead field matrix.

A second important constraint may be derived from fMRI indices of activation that enter as the constraint $\alpha$, a leading diagonal matrix with elements that reflect the prior probability of whether the source is active (here we allow only values of 0 , the variance is left unchanged, or 1 , the variance is increased according to the value of an hyperparameter). Combining these components, we obtain the following general expression:

$$
\begin{gathered}
\mathbf{H}_{\mathrm{B}}^{t} \mathbf{H}_{\mathrm{B}}=\left(\mathbf{B}^{t} \mathbf{C}_{\mathbf{j}} \mathbf{B}\right)^{-1} \\
\mathbf{C}_{\mathbf{j}}=\beta_{1} G^{t / 2} \mathbf{D} \mathbf{D G}^{1 / 2}+\beta_{2} \operatorname{diag}\left(\mathbf{L}^{t} \mathbf{L}\right)^{-1}+\beta_{3} \alpha+\cdots
\end{gathered}
$$

This formulation, in which the prior covariances are some linear combination of covariance components (structural, depth, and functional), is important because the hyperparameters $\beta$ can be estimated using iterative techniques such as the EM algorithm (Dempster et al., 1977). This will be pursued in a subsequent paper.

In the present case, a slightly modified approach was followed and $\mathbf{C}_{\mathbf{j}}$ was defined by

$$
\mathbf{C}_{\mathbf{j}}=\operatorname{diag}\left(\mathbf{L}{ }^{t} \mathbf{L}\right)^{-1}\left(\mathbf{I}_{N_{j}}+\boldsymbol{\beta} \boldsymbol{\alpha}\right)^{2}
$$

By ignoring the "hard" constraints associated with $\beta_{x}$ in Eq. (22) and combining the depth and fMRI priors, we were able to focus on the role of fMRI priors using a single hyperparameter $\beta$ (cf. Liu et al. (1998)). $\beta$ was assigned three values, 0 , 1 , and 4 , corresponding to no, weak, and strong (fMRI) location priors. The ensuing solutions will be refered to as "without priors" (or "wp0"), "with weak priors" (or "wpl"), and "with strong priors" (or "wp4"). This simplification can easily be interpreted as defining the weighting matrix $H$ that would be used to constrain the weighted norm of $\mathrm{j}$ (see Minimum Norm Solution) by

$$
\left(\mathbf{H}^{t} \mathbf{H}\right)^{-1}=\operatorname{diag}\left(\mathbf{L}^{t} \mathbf{L}\right)^{-1}\left(\mathbf{I}_{N_{j}}+\beta \boldsymbol{\alpha}\right)^{2} .
$$

By taking $\beta$ equal to 1 or 4 , we are actually assuming that the variance $\sigma^{2}$ of the a priori active location (defined by $\boldsymbol{\alpha}$ ) is 4 or 25 times larger than those of the other locations (if the depth constraint was discounted). 
FIG. 1. Successive steps of MRI processing. The structural MR image is segmented into the white and gray matter volumes. The dipoles are placed within the smoothed gray matter volume and the gradient of the anisotropically smoothed white matter volume is sampled at the dinole locations, providing a distribution of oriented dinoles.

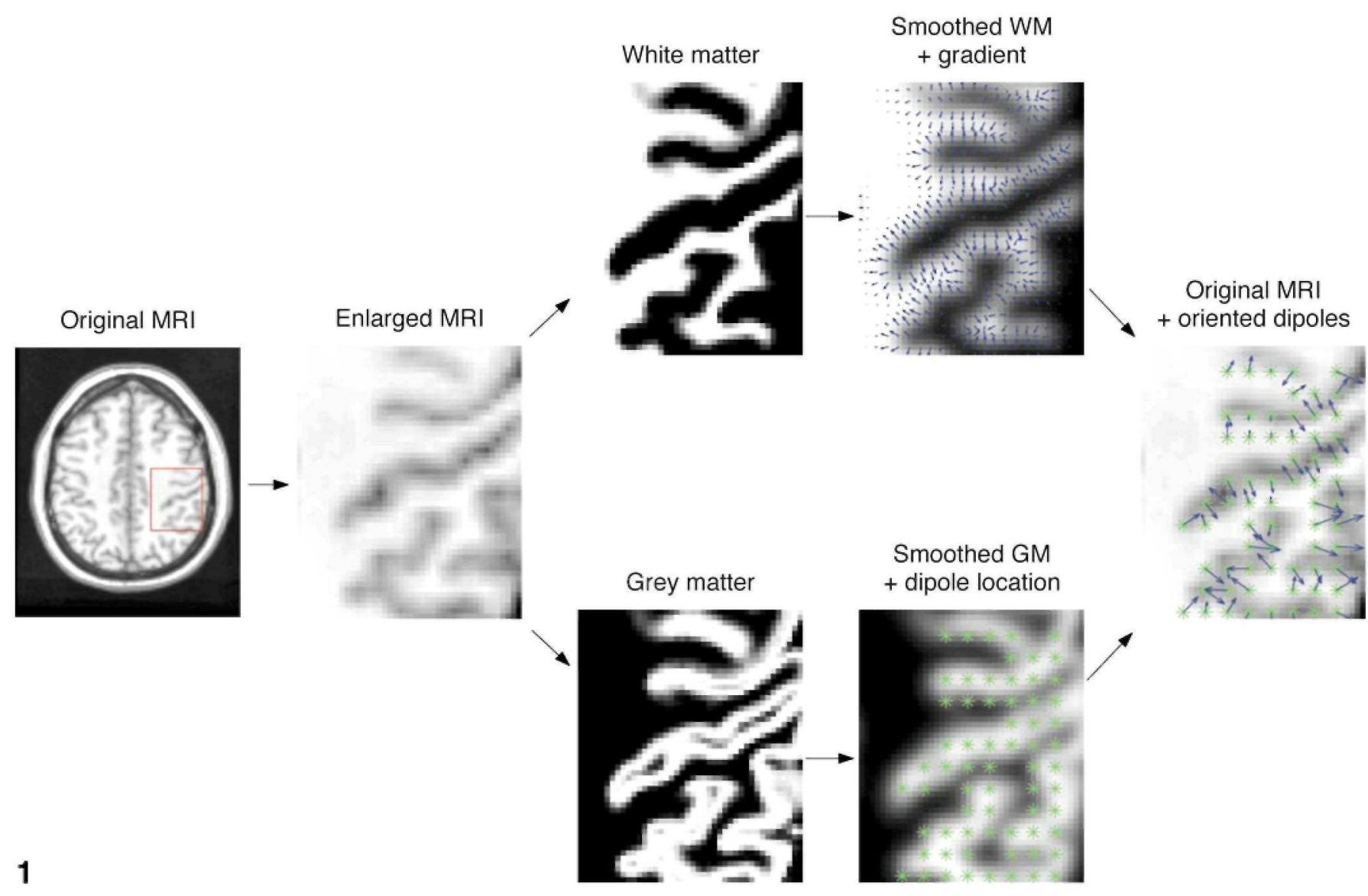

FIG. 2. Spectrum, on a logarithmic scale, of the normalized eigenvalues of the variance-covariance matrix $C_{i}$ $=G^{t / 2} D^{t} D G^{l / 2}$, for the two different spatial coherences considered: $\sigma=10 \mathrm{~mm}$ (top) and $\sigma=5 \mathrm{~mm}$ (bottom).
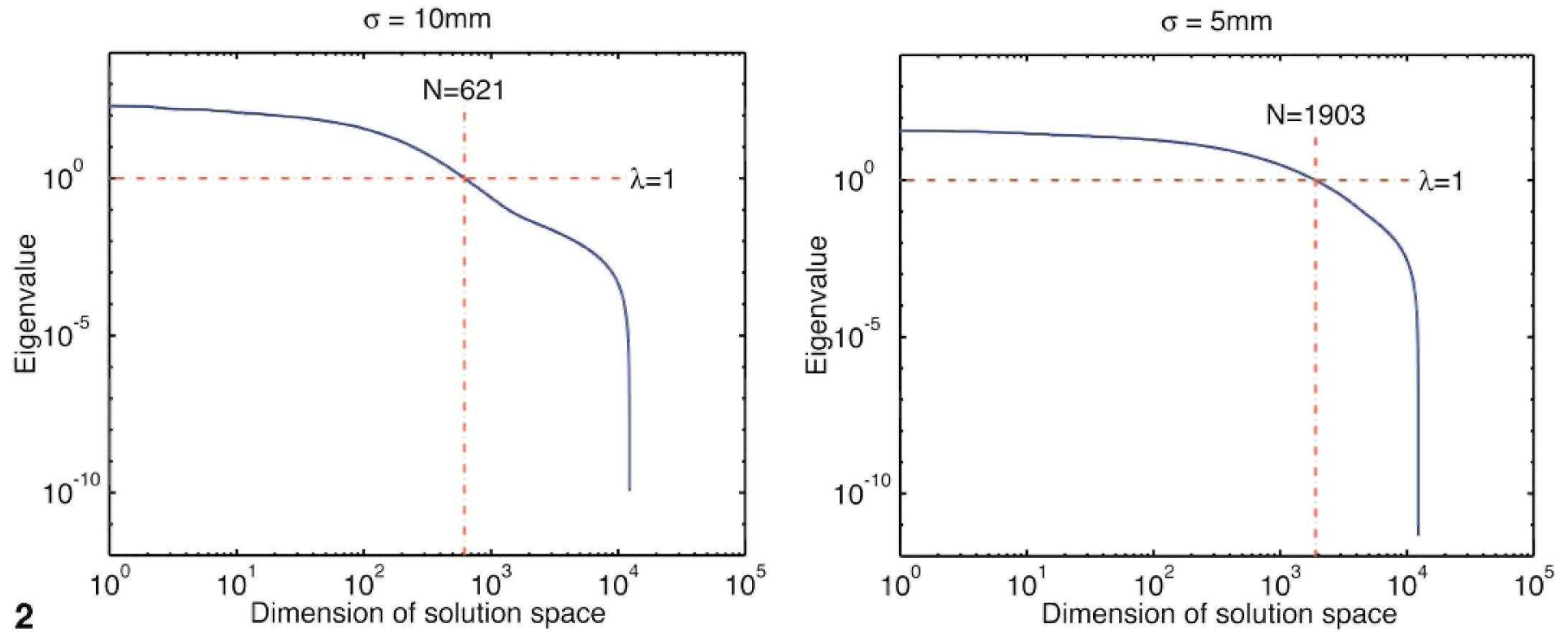
FIG. 3. Example of a single active source reconstruction. The original source set is shown at the top. Below are the source reconstructions (absolute values) obtained with the four solutions presented (IBF 10, IBF5, MS, and $W M N)$ without location priors. The amplitude of the sources was normalized between 0 and 1 for all the solutions.

Original source
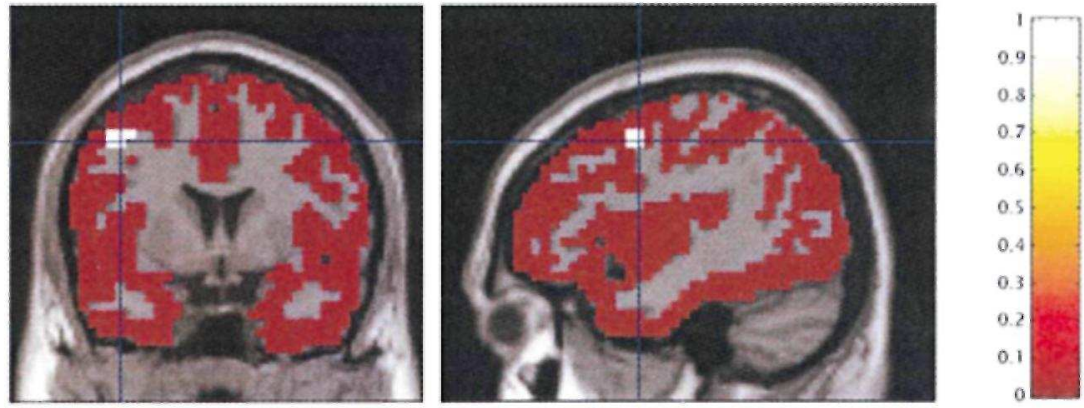

IBF10 source re-

construction
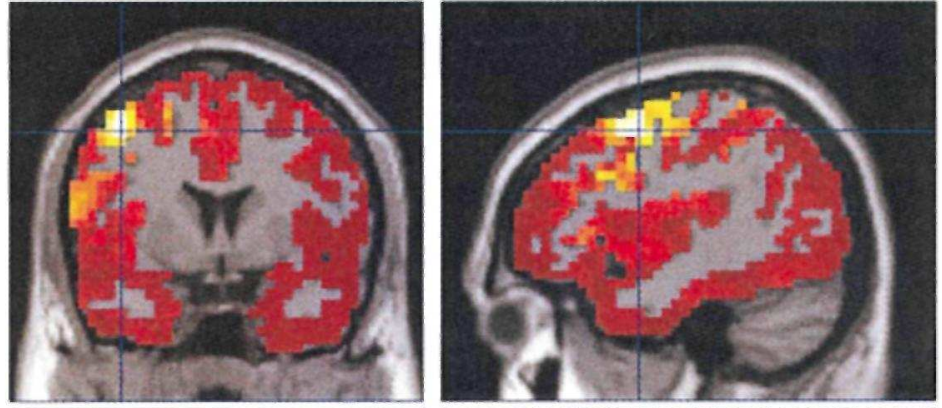

IBF5 source re-

construction
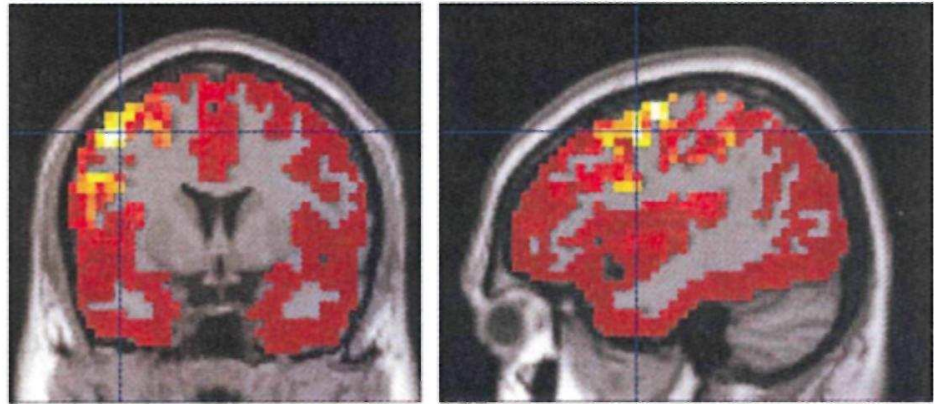

MS source re-

construction
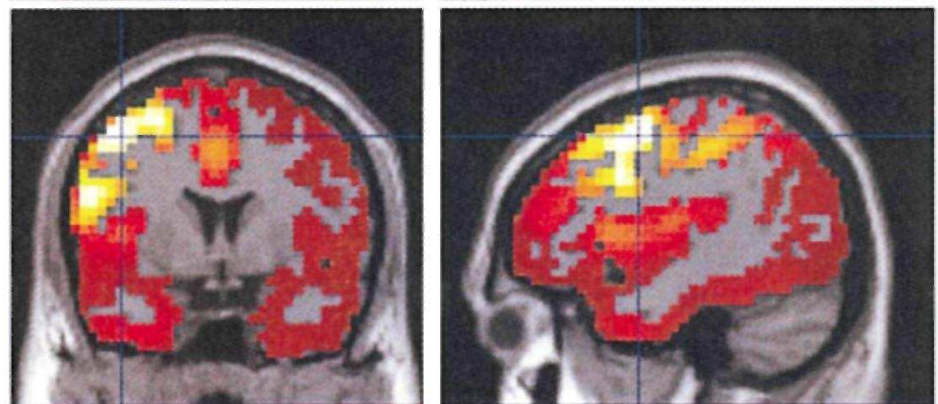

WMN source re-

construction
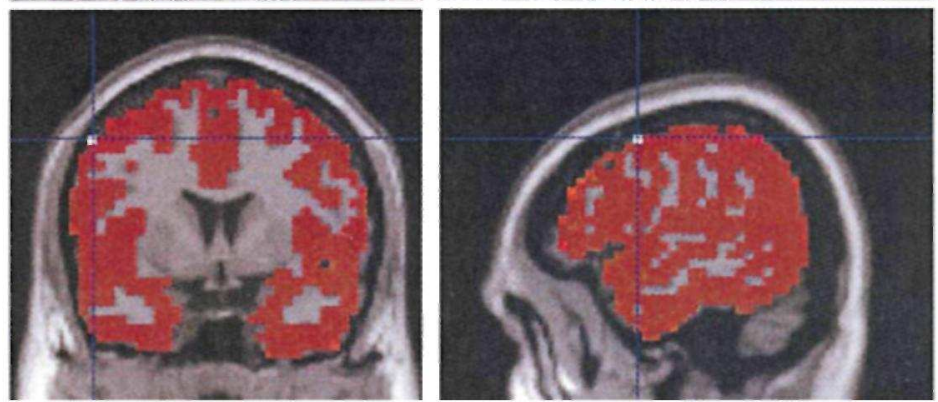


\section{Comparison with Other Methods}

The IBF approach described here was compared with two other commonly employed approaches: a weighted minimum norm solution and a maximum smoothness solution.

The WMN solution is simply the solution of the problem formulated in Eq. (13) given by Eq. (18) where the matrix $\mathbf{B}$ is the identity matrix and so $\mathbf{k}=\mathbf{j}$. In the version of the method employed here, the orientation of the dipoles was fixed as with the IBF method, so that only the amplitudes were unknown. The weighting matrix $\mathbf{H}$ was the same as the IBF simulations and is defined as in Eq. (24): it embodied the depth weighting and prior knowledge of the location of active sources. The WMN was used here to calculate three different solutions with the same range of $\beta$ : without priors, with weak priors, or with strong priors.

The MS solution is also a particular case of Eq. (13) and (18). The matrix B was again considered the identity matrix; i.e., $\mathrm{k}=\mathrm{j}$, but the orientations of the dipoles were left free. Strength parameters, $j_{x, i} j_{y, i}$, and $j_{z, i}$, were estimated for three independent and orthogonal dipoles, oriented along the three main axes $\vec{e}_{x}$,

$\vec{e}_{y}$, and $\vec{e}_{z}$, at each source location $i$ such that $\vec{j}_{i}=\left[j_{x, i}, j_{y, i}, j_{z, i}\right]$ and $\mathbf{j}_{f}=\left[\vec{J}_{1}^{t} \vec{J}_{2}^{t} \ldots \vec{J}_{N_{d i p}}^{t}\right]^{t}$. A single value $j_{i}$ for the amplitude of the electrical activity at each source location $i$ was then obtained by calculating the norm of the resulting dipole $j_{i}=\sqrt{j_{x, i}^{2}+j_{y, i}^{2}+j_{z, i}^{2}}$ Theweighting matrix $\mathrm{H}$ was defined as a weighted three-dimensional Laplacian, $\mathrm{H}=\mathrm{MW}$. W is a leading diagonal matrix defined by $\mathrm{w} \otimes\left[\begin{array}{lll}1 & 1 & 1\end{array}\right]^{\mathrm{t}}$, where $\mathrm{W}=\left[\left(\mathbf{I}_{N j}{ }^{\circledR}\left[\begin{array}{llll}1 & 1 & 1\end{array}\right]\right) \operatorname{diag}(\mathrm{L}\right.$ $\left.\left.{ }_{f}^{t} \mathrm{~L}_{f}\right)\right]^{1 / 2}$, which is equivalent to depth weighting used for the IBF and WMN solutions. The operator ${ }^{\circledR}$ denotes the Kronecker product and $\mathrm{L}_{f}$ is the $N_{e} \mathrm{x} N_{3 N j}$ lead field matrix corresponding to the orientation-free sources vector $\mathbf{j}_{f}$. The Laplacian matrix $\mathbf{M}$ is a regularized discrete three-dimensional second-order derivative operator defined as in Pascual-Marqui (1999). This method does not provide means to include further priors and thus only one solution was computed.

In short the only difference between the IBF and the WMN solutions was the use of spatial basis functions to constrain the solution space. The MS appoach can be considered an alternative implementation of spatial constraints through ensuring smoothness. The MS solution embedded the same depth priors but, unlike the IBF and $\mathrm{WMN}$, does not accomodate functional priors.

\section{RESULTS AND DISCUSSION}

Two criteria were used to assess and compare the performance of the different methods presented in the previous sections (IBF5, IBF10, WMN, and MS solutions). (1) "Localization error" (LE) is defined as the distance between the location $\vec{r}_{r}$ of the maximum (of the absolute value) of the reconstructed source distribution $\mathbf{j}_{\mathrm{r}}$ and the location $\vec{r}_{o}$ of the original source set $\mathbf{j}_{o}$ :

$$
\mathrm{LE}=\left|\vec{r}_{r}-\vec{r}_{o}\right| \text {. }
$$

(2) "Root mean square error" (RMSE) is defined as the norm of the difference between the reconstructed source distribution $\mathbf{j}_{\mathrm{r}}$ and the original source set $\mathbf{j}_{o}$,

$$
\operatorname{RMSE}=\sqrt{\sum_{I=1}^{N_{j}}\left(\frac{j_{r, I}}{\max \left(\operatorname{abs}\left(\mathbf{j}_{I}\right)\right)}-\frac{j_{o, 1}}{\max \left(\operatorname{abs}\left(\mathbf{j}_{o}\right)\right)}\right)^{2}},
$$

where $\mathbf{j}_{o, 1}$ is the 1 th element of $\mathbf{j}_{o}$.

The LE provides a measure of the localization accuracy of the reconstruction method; a small value of LE indicates that the location of the original source was recovered well. The RMSE measures the "goodness of fit" of the reconstruction; a small value of RMSE indicates a small discrepancy between the original and the reconstructed source distributions. The RMSE is useful only to further compare two solutions that have approximately the same LE. Indeed, if both solutions have almost the same LE, the one with the smaller RMSE would be prefered as the reconstructed source is then more focal. A very focal reconstructed source with a large LE will have a smaller RMSE than a blurred reconstructed source with a small LE, but the latter solution, although oversmoothed, provides at least some location information.

The sources $\mathbf{j}_{o}$ were generated at randomly selected locations within the head model described under Head and Source Model. Each source comprised a set of connected dipoles within a 7-mm radius of a "central" dipole. These constitute locally distributed sources. For each source $\mathbf{j}_{o}$, the potential at the electrodes was generated with Eq. (5). Information about prior location was provided as a sphere of 12-mm radius. Dipoles within this volume were defined as being a priori active sources; i.e., the corresponding diagonal elements of $\boldsymbol{\alpha}$ (see step 2: 
Minimum Norm Solution) were set to 1. Our simulations involved changing the strength of the functional priors and their accuracy in all combinations. We describe simulations of one focal source and proceed to simlutions of multiple coactive sources.

\section{Simulations with a Single Source}

The case of single active sources is considered first. For each source set, LE and RMSE were calculated for the IBF5, IBF10, and WMN methods in each of the following cases: (1) without priors, (2) with priors (weak and strong) centered on the original source (accurate priors), and (3) with priors (weak and strong) placed anywhere in the volume (incorrect priors). As presented under Comparison with Other Methods, no prior about the location of the sources can be included in the MS method; therefore the same solution was used for all the simulations. The results of a typical simulation are shown in Fig. 3.

\section{Solution without Location Priors}

The whole solution space was evaluated by generating a source set around each of the 12,300 dipoles in the model. The results obtained for LE are summarized in Fig. 4, and the mean RMSEs can be found in the second part of Table 1 (no priors, no mislocation). The IBF10, IBF5, and MS solutions have approximately the same localization accuracy, with about $80 \%$ of the sources recovered within $20 \mathrm{~mm}$ of their original location. The LE of the WMN solution is spread over a much larger range, and thus, although its RMSE is

smaller than that of any other method, its poor localization ability makes it a less useful method. The RMSE of the MS solution is on average larger than those of the IBF5 and IBF10 solutions. This results reflects the fact that the MS solution is overly smoothed. The IBF5 and IBF10 solutions are able to reconstruct focal activity more accurately. Because less coherence is imposed on the IBF5 solution, it is less "blurred" and thus the IBF5 solution yelds somewhat smaller RMSEs than the IBF10 solution.

\section{Solution with Accurate Location Priors}

Because of computational limitations it was not possible to assess the entire solution space with a priori location information (a new solution must be calculated for every set of priors). Therefore a set of 100 randomly selected sources were employed in these simulations. (For this reason, the results obtained for the MS solution are slightly different from those obtained in the previous section). The results for LE with weak and strong priors are summarized in Fig. 4 and the mean RMSEs can be found in the second part of Table 1 (no mislocation, weak and strong priors).

The inclusion of prior location information greatly improved the performance of the IBF5, IBF10, and WMN solutions, which all outperformed the MS solution. The IBF5 solution performed best of all, but both IBF solutions outperformed the WMN solution, particularly when the prior information was entered as a weak constraint only. With the IBF solutions, the reconstructed activity was more focal (smaller RMSE) and more than $80 \%$ of the sources were recovered within $4 \mathrm{~mm}$ of their original location.

\section{Solution with Incorrect Location Priors}

A set of 100 locations were randomly selected to provide prior location information. Corresponding IBF10, IBF5, and WMN solutions were then produced for the two levels of location constraint (weak and strong). Independently, 200 source sets $\mathbf{j}_{o}$ were randomly generated and their corresponding electrode potentials calculated. For every combination of prior location and original source $\mathbf{j}_{o}$, the source distribution $\mathbf{j}_{\mathrm{r}}$ was then reconstructed and the LE and RMSE were calculated.

The prior mislocation was defined as the distance between the location of the original source set and the corresponding prior information. The prior mislocation was divided into four "bands" of 30-mm width: 1-30, 31$60,61-90$, and 91-120 mm. Within each band of prior mislocation, a "maximum LE" was calculated, such that at least $80 \%$ of the sources were recovered within this LE (for simplicity, the LE was also divided in bands: 0-4, 4$12,12-20 \mathrm{~mm}, \ldots$ ). The mean RMSE for each band of prior mislocation was calculated also. For reference, the results obtained in the two previous sections are included in Table 1.

With weak priors, the LE of both IBF 10 and IBF5 solutions were similar to the case where no priors were employed. Thus the prior mislocation had rather little effect on the solution. In contrast, with strong priors, the IBF solutions were substantially affected by the incorrect prior location. This effect was greater for the IBF5 solution than for the IBF 10 solution with the difference being expressed mainly for smaller prior mislocations. In general the WMN solution behaved poorly.

\section{Simulations with Two Simultaneously Active Sources}

Here we consider the case of two simultaneously active source sets of equal strength. Two factors influence the reconstruction of two sources: the distance between them and their relative power in measurement space (i.e., at 
the electrodes). The power of a source set (at the electrodes) is calculated from the sum of squares of the electric potential generated at the electrodes. The power of proximate sources is very similar because their leadfields are almost colinear (although the orientation of the sources can still have some influence on the power of the electric field at the electrodes) and they might be difficult to distinguish in the reconstruction because of their proximity. Distant sources should be more easily distinguishable but their relative power can vary widely depending on their relative depth in the brain and their orientation, rendering their localization more difficult.

The methods presented in the previous sections (IBF5, IBF10, WMN, and MS solutions) were applied to simulated data using different ranges of source separation and power. The separation was divided into five "bands" of 30-mm width (as in Solution with Incorrect Location Priors for the prior mislocation): 1-30, 31-60, 61-90, and 91-120 $\mathrm{mm}$. The ensuing relative power of source pairs was used to further stratify the source configurations into five "bands" according to a logarithmic scale: $10^{0.0}-10^{0.1}, 10^{0.1}-10^{0.2}, 10^{0.2}-10^{0.3}$, and $10^{0.3}-10^{0.4}$ ,ie, 1.00-1.26, 1.26-1.58, 1.58-2.00, and 2.00-2.51. One combination of separation and relative power, separation of 1-30 mm and relative power 2.51-3.16, was not possible to achieve, and in this case no results are shown. For each of the other combinations, 100 pairs of sources were selected randomly, data were generated, and the activity was reconstructed.

The RMSE was calculated with Eq. (26). As two sources are employed in these simulations, the LE was calculated as follows. For each pair of sources $\mathbf{j}_{o 1}$ and $\mathbf{j}_{o 2}$, centered at location $\mathbf{r}_{1}$ and $\mathbf{r}_{2}$, the original source set $\mathbf{j}_{o}$ was the sum of $\mathbf{j}_{o 1} /\left\|\mathbf{j}_{\text {o1 }}\right\|$ and $\mathbf{j}_{o 2} /\left\|\mathbf{j}_{\text {o2 }}\right\|$, and the potential at the electrodes was then generated using Eq. (5). The absolute value of the reconstructed activity $\mathbf{j}_{\mathrm{r}}$ (for the MS solution, the 2-norm of the three components at each location was used instead of the absolute value) was thresholded at $50 \%$ of its maximum, leaving a set of suprathreshold clusters of active sources. The location $r_{b}$ of the maximum of each cluster (cluster peak) was compared to $r_{1}$. and $r_{2}$. According to the number and location of the maxima, three cases are possible: (1) only one peak surviving after thresholding (in this case only one value can be attributed to the LE, the number of original sources recovered (NRec) is 1 , and the number of spurious reconstructed sources (NS-pur) is 0), (2) many peaks $(\geq 2)$ but all closer to $\mathbf{r}_{1}$ than $\mathbf{r}_{2}$ (surviving now only one value (the minimum distance between $r_{b}$ and $\mathbf{r}_{1}$ can be attributed to the LE, NRec is 1 , and NSpur is equal to the number of clusters minus 1), and (3) many peaks $(\geq 2)$ that are spread around $\mathbf{r}_{1}$ and $\mathbf{r}_{2}$ surviving (here two values (the minimum distance between the $r_{b}$ 's and $\mathbf{r}_{1}$ and $\mathbf{r}_{2}$ ) can be attributed to the LE, NRec is 2 , and NSpur is equal to the number of clusters minus 2).

In addition to the LE and the RMSE criteria, the number of original sources recovered (NRec) and the number of spurious reconstructed sources (NSpur) were assessed for each reconstruction method. NRec provides the number of sources actually recovered while NSpur indicates how many spurious (and difficult to interpret) clusters are left after thresholding. An example of the reconstructed activities is presented in Fig. 5. 
FIG. 4. Localization error (LE) for the four solutions applied to the simple source simulated data, with and without prior location: none (top), weak (middle), and strong (bottom). The MS solutions do not incorporate prior location information.

\section{No priors}

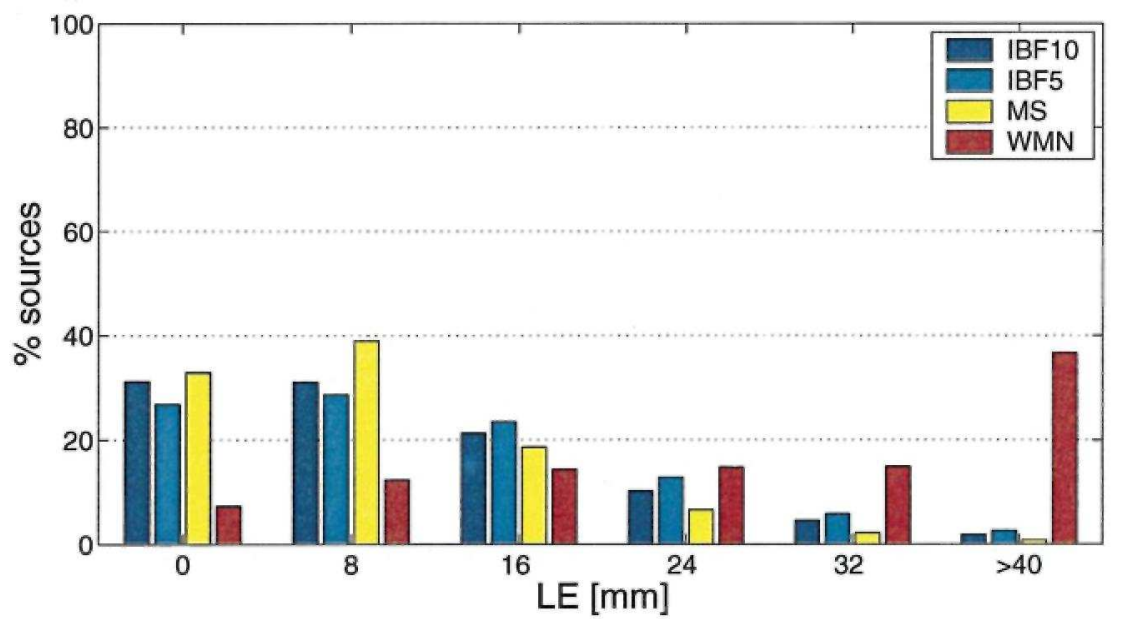

Weak priors

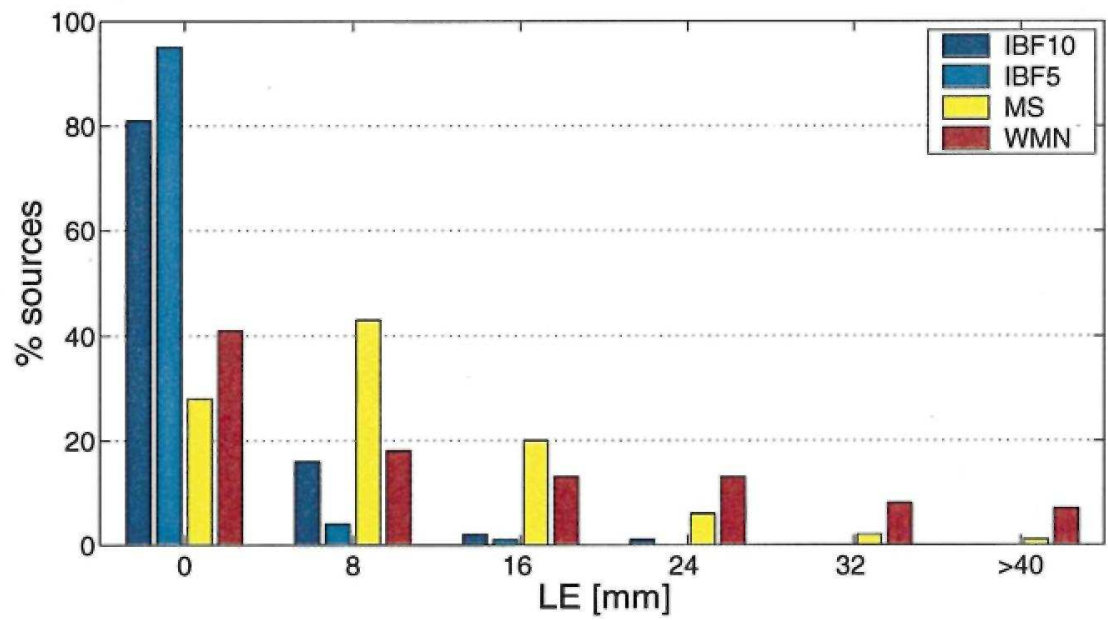

\section{Strong priors}

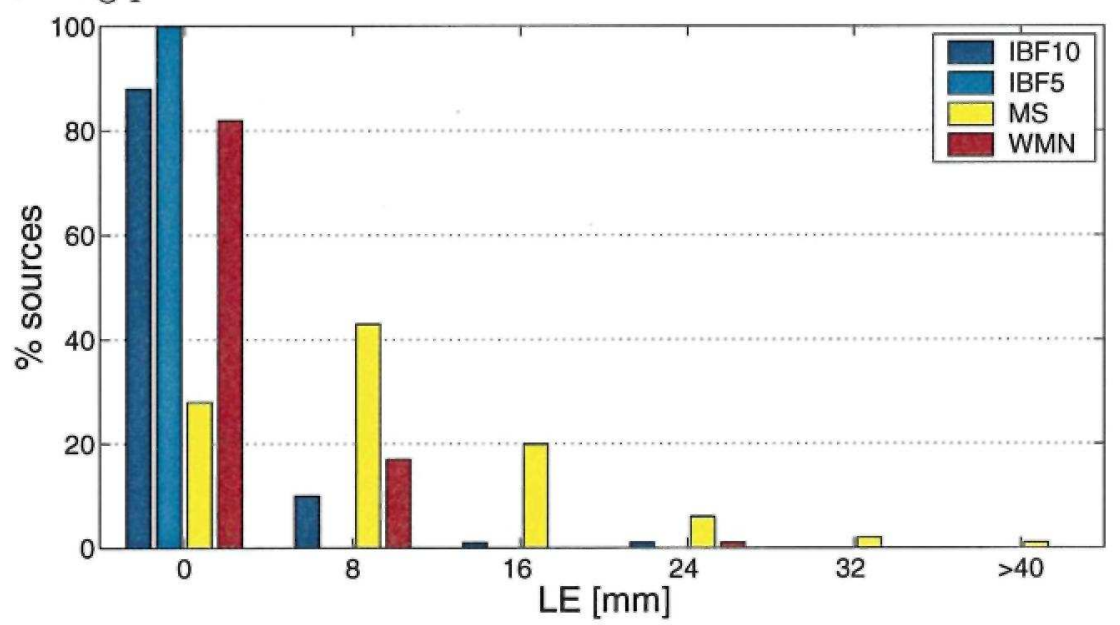


FIG. 5. Example of reconstructions of two simultaneously active sources. The original source sets are shown at the top. Below are the source reconstructions (thresholded absolute value) obtained with the four solutions presented (IBF10, IBF5, MS, and WMN) without location priors. The amplitude of the sources was normalized between 0 and 1 for all the solutions.

Original source
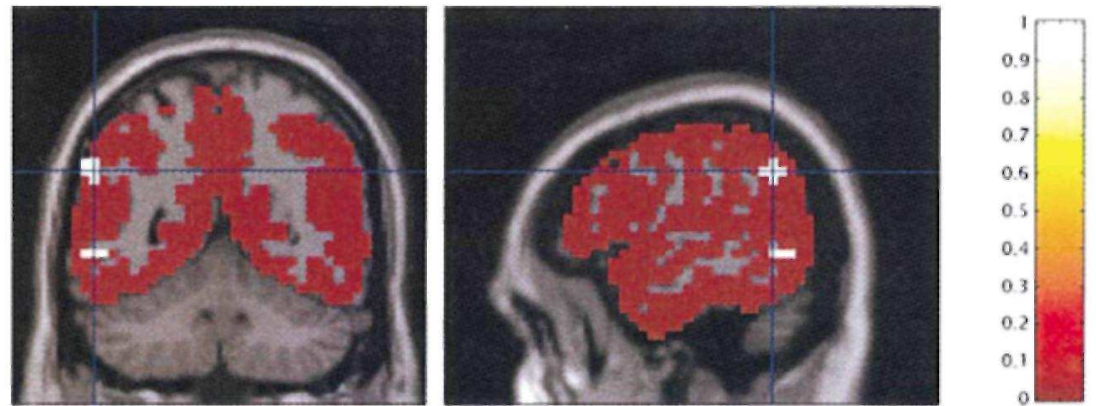

IBF10 source re-

construction
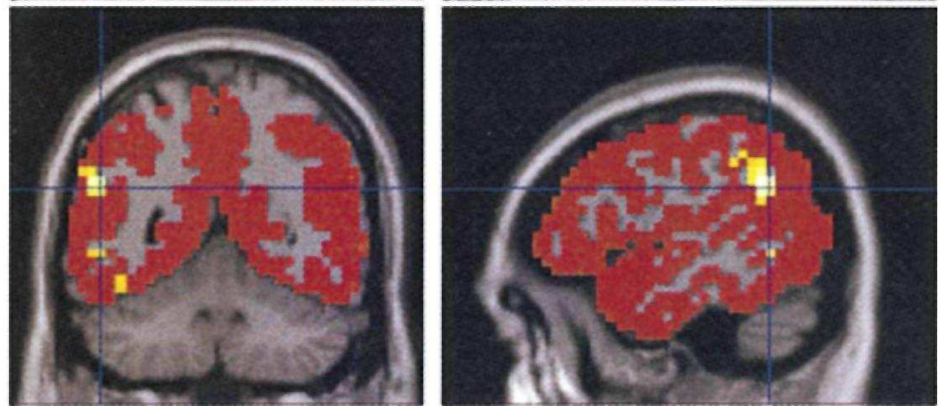

IBF5 source re-

construction
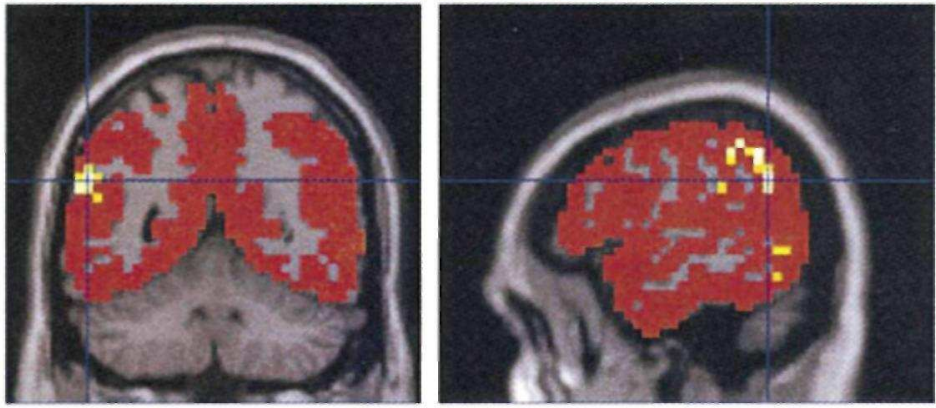

MS source
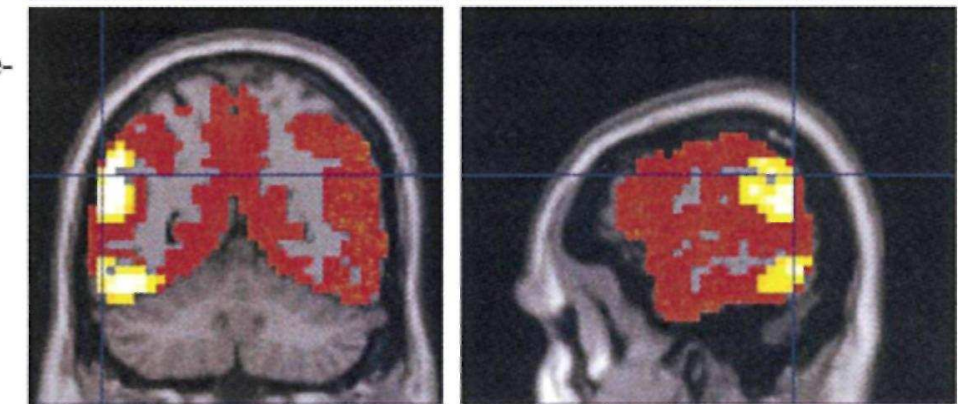

construction
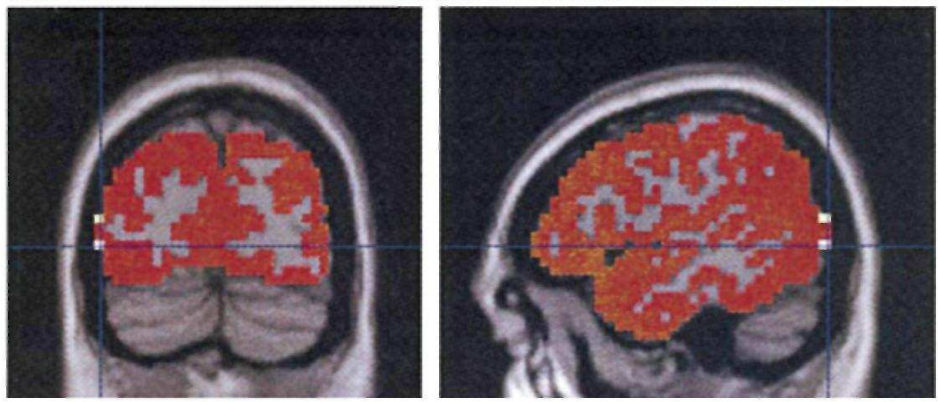

WMN source re-

construction 


\section{Solution without Location Priors}

Here the solutions were calculated without prior knowledge about the location of active sources. The number of original sources recovered varied considerably among the methods and for the different degrees of source separation and relative power. The values of NRec are summarized in Table 2.

For every method and source separation, the risk of recovering only one source $(\mathrm{NRec}=1)$ increased with the relative power of the sources. Overall, the two sources were recovered in only 48, 47, 40, and 56\% of the cases for the IBF10, IBF5, MS, and WMN solutions, respectively. The values of LE, NSpur, and RMSE seemed to depend mainly on the number of sources recovered $(\mathrm{NRec}=1$ or $\mathrm{NRec}=2)$ and varied little according to the distance between sources or their relative power. Therefore, the values of NSpur and RMSE were averaged over all conditions, separately for $\mathrm{NRec}=1$ or $\mathrm{NRec}=2$. The LE was also averaged this way but, as under Solution with Incorrect Location Priors, a "maximum LE" was calculated such that at least $80 \%$ of the sources were recovered within this LE.

When NRec $=2$, two values were available for LE, a "small" one and "large" one. As the order of the sources within each pair is arbitrary, two "maximum LEs" were calculated, one for the "small LE" (best case) and one for the "large LE" (worst case). These results are summarized in Table 3.

The maximum LEs obtained were smaller than those observed for single sources (compare the first line of Table 1 with Table 3, especially for the cases of NRec $=1$ and NRec $=2$ for the "small" LE). The difference is due to the way that the LE is measured. Whereas previously (Solution without Location Priors and Solution with Accurate Location Priors) only the maximum of the absolute value of the reconstructed activity was used, here maxima of lesser amplitude were also considered.

As the smoothness of the IBF solutions is less than that of MS solution, close sources can be better distinguished with the former method. The IBF solutions were less likely to miss a source but also produced more spurious active sources than the MS solution. This renders the interpretation of the reconstructed activity more difficult (even if the clusters are generally spread around the location of the original sources) but a higher threshold could be used (with the risk of missing an original source). The small value of NSpur $(<1)$ for the MS solution when only one source is recovered shows that in many cases a single cluster was left after thresholding, but there were still a few cases where there was spurious reconstructed activity.

The figures obtained for the RMSE correspond to those observed for single sources; see top row of Table 1. The RMSE is slightly larger than those in the previous simulations but this can be explained by the fact that there were two active sources instead of one. 
TABLE 1: LE and RMSE for Different Degrees of Mislocation of the Prior Information

\begin{tabular}{|c|c|c|c|c|c|}
\hline & Priors mislocation & Priors strength & IBF10 & IBF5 & WMN \\
\hline \multirow[t]{7}{*}{$\operatorname{Max} \operatorname{LE}(\mathrm{mm})$} & \multirow[t]{3}{*}{ No mislocation } & No & $20(86 \%)$ & $20(85 \%)$ & $44(85 \%)$ \\
\hline & & Weak & $4(90 \%)$ & $4(95 \%)$ & $28(85 \%)$ \\
\hline & & Strong & $4(97 \%)$ & $4(100 \%)$ & $4(82 \%)$ \\
\hline & \multirow[t]{2}{*}{$1-30 \mathrm{~mm}$} & Weak & $20(86.3 \%)$ & $20(80.2 \%)$ & $44(84.2 \%)$ \\
\hline & & Strong & $20(81.8 \%)$ & $28(95.8 \%)$ & $44(85.5 \%)$ \\
\hline & \multirow[t]{2}{*}{$>30 \mathrm{~mm}$} & Weak & $20(84.6 \%)$ & $20(83.0 \%)$ & $44(83.5 \%)$ \\
\hline & & Strong & $20(83.9 \%)$ & $28(82.5 \%)$ & $44(82.7 \%)$ \\
\hline \multirow[t]{7}{*}{ Mean RMSE } & \multirow[t]{3}{*}{ No mislocation } & No & 11.0 & 10.3 & 3.6 \\
\hline & & Weak & 8.2 & 5.0 & 4.7 \\
\hline & & Strong & 6.5 & 2.7 & 3.8 \\
\hline & \multirow[t]{2}{*}{$1-30 \mathrm{~mm}$} & Weak & 11.0 & 9.5 & 3.6 \\
\hline & & Strong & 10.8 & 8.5 & 3.6 \\
\hline & \multirow[t]{2}{*}{$>30 \mathrm{~mm}$} & Weak & 11.0 & 9.7 & 3.6 \\
\hline & & Strong & 10.9 & 9.1 & 3.6 \\
\hline
\end{tabular}

Note. For mislocation greater than $30 \mathrm{~mm}$, the maximum LE and mean RMSE varied little and only their average values are calculated. For comparison the values obtained for the MS solution are the following: within a LE of $20 \mathrm{~mm}, 91 \%$ of the sources were recovered and the mean RMSE was 16.2

TABLE 2: Percentage of Source Reconstructions Where Both Sources Were Recovered (NRec = 2)

Source separation $(\mathrm{mm})$

\begin{tabular}{lllllllll}
$\begin{array}{l}\text { Relative } \\
\text { power }\end{array}$ & $1-30$ & \multicolumn{3}{c}{$31-60$} & $61-90$ & \multicolumn{3}{c}{$91-120$} \\
\hline $10^{00}-10^{01}$ & 78 & 84 & 62 & 56 & 56 & 50 & 65 & 58 \\
& 39 & 66 & 53 & 69 & 51 & 66 & 63 & 67 \\
$10^{01}-10^{02}$ & 74 & 76 & 53 & 53 & 51 & 49 & 28 & 25 \\
& 36 & 68 & 51 & 62 & 51 & 68 & 37 & 42 \\
$10^{02}-10^{03}$ & 72 & 75 & 46 & 39 & 40 & 41 & 33 & 39 \\
& 41 & 58 & 38 & 54 & 40 & 55 & 37 & 42 \\
$10^{03}-10^{04}$ & & n.a. & 35 & 36 & 30 & 24 & 18 & 18 \\
& & & 27 & 54 & 22 & 44 & 20 & 39
\end{tabular}

Note. In each cell of the table, the values in the upper left and upper right quadrants correspond to the IBF10 and IBF5 solutions, respectively, and the values in the lower left and lower right quadrants correspond to the MS and WMN solutions, respect 
TABLE 3: Max LE and Mean Values of NSpur and RMSE When NRec $=1$ or NRec $=2$

\begin{tabular}{llllll} 
& IBF10 & & IBF5 & MS & WMN \\
\hline max LE $(\mathrm{mm})$ & NRec $=1$ & $12(96 \%)$ & $12(98 \%)$ & $12(82 \%)$ & $28(82 \%)$ \\
& NRec $=2$ & $12(98 \%)$ & $12(99 \%)$ & $12(92 \%)$ & $28(87 \%)$ \\
& $20(88 \%)$ & & $20(90 \%)$ & $20(83 \%)$ & $44(81 \%)$ \\
Mean NSpur & NRec $=1$ & 2.38 & 5.12 & 0.53 & 1.83 \\
& NRec $=2$ & 5.50 & 10.83 & 1.25 & 4.39 \\
Mean RMSE & NRec $=1$ & 9.93 & 9.18 & 14.68 & 4.31 \\
& NRec $=2$ & 12.94 & 12.01 & 18.51 & 4.65 \\
\hline
\end{tabular}

TABLE 4: Percentage of Reconstructed Sources Where Both Sources Were Recovered $(N R e c=2)$

Source separation $(\mathrm{mm})$

\begin{tabular}{lllllllll}
\cline { 2 - 8 } $\begin{array}{l}\text { Relative } \\
\text { power }\end{array}$ & $1-$ & -30 & 31 & -60 & $61-$ & -90 & $91-$ & -120 \\
\hline $10^{0.0}-10^{0.1}$ & 61 & 52 & 53 & 56 & 54 & 55 & 63 & 64 \\
& 52 & 62 & 50 & 65 & 51 & 65 & 57 & 72 \\
$10^{0.1}-10^{0 . .2}$ & 55 & 55 & 38 & 52 & 33 & 47 & 44 & 58 \\
& 47 & 55 & 43 & 61 & 41 & 58 & 26 & 42 \\
$10^{0.2}-10^{0.3}$ & 52 & 43 & 32 & 34 & 39 & 39 & 41 & 43 \\
& 53 & 56 & 26 & 42 & 38 & 45 & 34 & 50 \\
$10^{0.3}-10^{0 . .4}$ & 49 & 42 & 27 & 32 & 29 & 43 & 37 & 53 \\
& & & 27 & 33 & 26 & 29 & 18 & 23 \\
& n.a. & & 29 & 32 & 20 & 35 & 21 & 31 \\
& & & 25 & 38 & 21 & 39 & 25 & 29
\end{tabular}

Note. In each cell of the table, the values in the top row correspond to the IBF10 solution with weak (left) and strong (right) priors, in the middle row to the IBF5 solution with weak (left) and strong (right) priors, and in the the bottom row to the WMN solution with weak (left) and strong (right) priors, respectively. 
TABLE 5: Max LE and Mean Values of NSpur and RMSE for NRec $=1$ and NRec $=2$

\begin{tabular}{|c|c|c|c|c|c|}
\hline & Priors & Nrec & IBF 10 & IBF5 & WMN \\
\hline \multirow[t]{6}{*}{ Max LE (mm) } & Weak & 1 & $4(92 \%)$ & $4(100 \%)$ & $12(86 \%)$ \\
\hline & & 2 & $4(97 \%)$ & $4(100 \%)$ & $4(94 \%)$ \\
\hline & & & $12(94 \%)$ & $4(98 \%)$ & $28(86 \%)$ \\
\hline & Strong & 1 & $4(98 \%)$ & $4(100 \%)$ & $4(98 \%)$ \\
\hline & & 2 & $4(100 \%)$ & $4(100 \%)$ & $4(99 \%)$ \\
\hline & & & $4(90 \%)$ & $4(100 \%)$ & $4(88 \%)$ \\
\hline \multirow[t]{4}{*}{ Mean NSpur } & Weak & 1 & 0.57 & 0.16 & 1.41 \\
\hline & & 2 & 2.22 & 0.72 & 3.34 \\
\hline & Strong & 1 & 0.161 & 0.01 & 0.76 \\
\hline & & 2 & 0.75 & 0.05 & 1.61 \\
\hline \multirow[t]{4}{*}{ Mean RMSE } & Weak & 1 & 7.27 & 5.08 & 5.34 \\
\hline & & 2 & 9.97 & 6.45 & 6.16 \\
\hline & Strong & 1 & 5.95 & 3.33 & 4.40 \\
\hline & & 2 & 7.76 & 3.45 & 4.77 \\
\hline
\end{tabular}

\section{Solution with Two Location Priors}

In this section, the same set of sources as those under Solution without Location Priors was used but the solutions (IBF5, IBF10, and WMN only) were calculated with (weak and strong) prior knowledge about the location of both active sources. Similarly to the case without priors (see Solution without Location Priors, the number of sources recovered (NRec) varied greatly between conditions (source separation and relative power) and type of solution. The values of NRec are summarized in Table 4.

For the IBF5 solution with strong priors, the proportion of cases where only one source of two was recovered was only slightly reduced, compared to the simulations without prior location (except for sources separated by less than $30 \mathrm{~mm})$. Otherwise the risk of recovering only one source $(\mathrm{NRec}=1)$ is larger when priors are included than without, as can be seen by comparing Tables 2 and 4 . The two sources are more often recovered with strong priors than with weak priors, especially for distant sources. Over all, two sources are recovered in 42, 38, and $34 \%$ of the cases with weak priors and in 43,50 , and $45 \%$ of the cases with strong priors for the IBF 10 , IBF 5 , and WMN solutions, respectively. The values of LE, NSpur, and RMSE vary little between conditions (source separation and relative power) but depend instead on the number of sources recovered $(\mathrm{NRec}=1$ or $\mathrm{NRec}=2$ ). Therefore, the values of LE, NSpur, and RMSE were averaged and presented as described in under Solution without Location Priors. These results are summarized in Table 5.

The inclusion of location priors greatly improved the LE, as was the case in the simulations with one active source described under Solution with Accurate Location Priors. With weak or strong priors, both IBF solutions reconstructed most of the sources within $4 \mathrm{~mm}$ of their original locations, except in the "worst case" of the IBF 10 solution with weak priors. The LE of the WMN solution was improved by the inclusion of weak priors but reached the level of the IBF solutions only with the use of strong priors. All these values are in agreement with those obtained for the simulations with only one active source; see top row of Table 1.

The number of spurious reconstructed sources was greatly reduced by the introduction of location priors. There was still some spurious reconstructed activity but much less than when no priors were included, as can be seen by comparing Tables 3 and 5. The largest benefit was obtained for the IBF5 solution. With strong priors, the IBF5 solution gave almost the ideal values of NSpur $=0$ for NRec $=1$ (one source is missing but there are no spurious sources) and NRec $=2$ (the two sources are recovered without spurious activity). The WMN solution showed significantly smaller NSpur only for the strong priors but these values were still worse than those of the IBF solution.

The RMSE values were also improved by the inclusion of priors and proved to be similar to those obtained when there was only one active source to reconstruct (compare Tables 3 and 5). 


\section{Summary of Results}

In the absence of prior information about location, the IBF solutions had approximately the same localization ability as the MS solution but the IBF's RMSE was smaller. Because less smoothness is imposed on the IBF solutions than on the MS solution, the former allow solutions with higher spatial frequencies and therefore the reconstructed activity for the IBF solutions were more focal. When two sources were active, the IBF solutions were more likely to recover both sources but the number of spurious sources was much larger for the IBF than for the MS solutions, especially if little smoothing was imposed. The level of smoothness imposed on the solution is the key factor affecting the regularization of the source localization problem. Clearly the IBF approach lies somewhere between an overly smoothed MS solution and an insufficiently constrained WMN solution.

When location priors were introduced the LE was greatly reduced, even with a weak constraint for the IBF solutions, but the WMN solution required a strong constraint to reach the level of performance attained by the IBF solutions. When two sources were considered the number of spurious sources was also greatly reduced, especially with strong priors, rendering the reconstructed activity more easily interpretable. However, the risk of missing one source was increased somewhat, except for the IBF solution with little smoothing and strong priors. Nevertheless, only the WMN solution with weak priors missed more sources than the MS solution. When the priors were included, the IBF solutions provided better reconstructions than the WMN solution, and the MS solution was outperformed in every case by the three other solutions. The combination of smoothing and location priors offers the best regularization of the source localization problem but, in relation to the case without priors, less smoothing seems to be required.

The case of mislocated priors does not impinge on the MS solution. With incorrect location priors, the performance of the IBF solution depended on the amount of smoothing imposed. With a large smoothing constraint, the results were similar to the case where no location priors were employed. In constrast the IBF solution with a small smoothing constraint was more affected by prior mislocation (especially when the prior location was strong). The larger smoothing constraint rendered the solution less sensitive to the bias produced by the mislocated prior. Therefore the combination of greater smoothing and weak location priors may be preferred when there is risk that the location prior is inaccurate.

In conclusion, the constraints afforded by informed spatial basis functions ensured that, overall, IBF outperformed the MS and WMN approaches (in the limiting case of these noise-free simulations).

\section{Discussion}

The only way to overcome the intrinsic limitations (on temporal or spatial resolution) of individual brain imaging modalities is to combine data obtained from different techniques within the same analytical framework. Such a combination should provide an optimal solution that harnesses the strengths of each technique.

In general, the advantages of a distributed linear approach to the source localization problem, as presented under The General Approach, are the following: a noniterative linear solution is readily available and calculable, as presented under Minimum Norm Solution. Unlike ECD approaches, no assumptions are made about the number of active sources, and sources can be relatively diffuse. In the framework of a distributed linear solution, structural information extracted from MR images, such as source orientation and cortical contiguity, can be combined with the EEG data. It is also possible to include other sources of data, such as activation maps from functional MRI (or PET) studies, to refine the localization without compromising the fine temporal resolution of EEG data.

Structural information is sometimes obtained for the EEG source constraints by reconstructing the cortical surface from an MR image. This is a nontrivial and difficult process (Dale and Sereno, 1993; Dale et al., 1999; Fischl et al, 1999; Fischl and Dale, 2000; Goebel and Max Planck Society, 2000) but, in the present work, the explicit reconstruction of the cortical surface is avoided by using a user-independent method to extract anatomical information from MR images. The sources are not constrained to lie on a folded surface but are spread on a fully three-dimensional grid. The orientation of each dipole is directly incorporated in the lead field matrix. Each oriented dipole can thus be viewed as a voxel in a three-dimensional volume, the amplitude of a dipole representing the activity in that voxel.

The approach adopted in the present paper does not strictly follow the scheme of a distributed linear reconstruction. The spatial IBF obtained from the anatomical information allows a two-step approach to imposing constraints on the source localization problem. Firstly, the size of the problem is reduced by projecting the solution space onto the subspace spanned by the spatial IBFs. Secondly, the anatomically constrained problem is solved. In this paper, a linear solution was chosen because of its useful properties and ease of use, but other methods could be employed to estimate the best combination of spatial basis functions. 
The extraction of spatial IBFs is the key element in the approach presented here. Although systematic, it has the disadvantage of being time consuming and computationally demanding. Fortunately, the spatial IBFs need only be calculated once for a specific head and source model. Afterward, various hypotheses about prior location of activation and different data sets can be studied with the same spatial IBF set but different "soft" constraints. The introduction of the spatial IBF thus offers a clear separation between the "hard" constraints relying on anatomy and other more intuitive "soft" constraints.

In the absence of prior location information, the MS solution provides source reconstruction with an accuracy similar to that of the IBF solution. But as its name suggests, the reconstructed sources may be too smooth. It is not possible to make full use of anatomical information; whereas the sources are constrained to the gray matter, their orientation is left free and the smoothness constraint, a simple three-dimensional Laplacian, does not respect cerebral anatomy. Moreover, no functional information about the (possible) location of the activity within the gray matter can be introduced.

The WMN solution, as employed here, uses only part of the structural information to constrain the solution: the orientations of the sources are fixed but no spatial correlation among them is imposed. In contrast to the MS solution, the WMN was inefficient without location priors but was capable of accurate localization with strong location priors. The solution presented and tested in Liu et al. (1998), here termed "Surface WMN" (SWMN), is equivalent to the WMN solution employed here. The difference between the two solutions lies in the source model: the sources are spread on the extracted cortical surface in Liu et al. (1998) instead of on a threedimensional grid as in the WMN employed here. In both WMN and SWMN, no spatial correlation

between the sources is assumed and a priori information about the location of the sources, obtained from fMRI activation maps, was introduced to better constrain the problem. The SWMN solution was tested by Liu et al. (1998) for four values of the relative weighting of the prior location (fMRI activation), 0, 90, 99, and 100\%, which corresponds, in Eq. (24), to values of $\beta$ equal to $0,2.16$, 9, and $\infty$. In our simulations three levels were used ( $\beta=0,1$, or 4 ), corresponding to relative weightings of 0,75 , and $96 \%$. By setting $\beta \rightarrow \infty$, one assumes an infinitely strong confidence in the location priors, effectively precluding any activity outside those priors. The decision of Liu et al. (1998) to use a compromise value of $90 \%$, i.e., $\beta=2.16$, for the weighting of the a priori source location is supported by the results of our simulations. Interestingly, the introduction of some smoothness in the IBF solution means that a smaller bias or weighting toward the a priori location can be employed, as good localization results are already obtained with the IBF solutions and $\beta=1$, unlike WMN. At this value of $\beta$, the solutions were little affected by location priors that were wrongly localized.

The solutions used here were tested with two simultaneously active sources in an extreme case where both sources are perfectly synchronized in time and have exactly the same strength in the source space, although not in the measurement space. Under those particular conditions and without location priors, the two sources were recovered efficiently (the WMN solution is not considered here) in less than $50 \%$ of the cases. Moreover there were always some spurious reconstructed sources (according to the amplitude thresholding adopted). Contrary to what might have been expected, the introduction of location priors did not improve the recovery of both sources. The location priors did, however, improve the solution by greatly reducing the number of spurious sources. If the signals produced by two sources are almost collinear in the measurement space, or if the amplitude of the signal generated by one source is larger than that generated by the other one, then one source will be generally "hidden" or "shadowed" by the other. Weak location priors cannot make the sources more "visible" but will help by locating the sources more focally.

The solutions presented here were not compared to ECD approaches. Apart from the moving-ECD solution, where the iterative fitting procedure has to simultaneously optimize the location, orientation, and amplitude of the ECDs, the fixed-ECD solutions are particular cases of the more general linear distributed solution. ECDbased attempts at localization have used fMRI (or PET) activation maps as prior constraints on the inverse problem: a single ECD is placed (or "seeded") at the center of gravity of fMRI (or PET) regions of activation, or at any location that seems useful, and then the orientation and amplitude of the ECDs are fitted to the EEG data, e.g., Opitz et al. (1999). In the linear solution framework, the seeded-ECD solution can be obtained by setting the fMRI weighting, parameter $\beta$ in Eq. (24), to infinity or equiv-alently by setting the a priori variance of the sources outside the fMRI activations to 0 .

We have attempted to provide a fairly comprehensive evaluation of the informed basis function approach, in relation to existing approaches. Within the parameters space covered by our simulations the informed basis approach out-performed alternatives. However, one cannot generalize these findings. The different approaches evaluated all adopt different constraints implicit in their respective cost functions. Different cost functions will be appropriate in different situations and under different assumptions about underlying sources that cause observed data. Our technique was developed under the assumption that these sources are distributed and conform to appropriate anatomical constraints. This is a requirement that we foresee will be important in the integration of 
EEG and fMRI data but may not be important in other applications in which a smoothness constraint or weighted minimum approach may be entirely sufficient.

The effect of errors in the solution of the forward problem on the accuracy of the reconstruction of the sources was not assessed here. There is no way to completely characterize this effect in a realistic head model as the synthetic data are generated with the same model (or lead field) used to solve the inverse problem. Although the methods described above apply to both electroencephalographic and magnetoencepha-lographic data, only EEG data were used to illustrate the approach in this article. The results obtained are directly and easily transferable to MEG data. When both EEG and MEG data are available simultaneously, they should be combined and used simultaneously to solve the source localization problem (Fuchs et al., 1998; Baillet et al, 1999) as the two kinds of data contain complementary information.

In conclusion we have demonstrated that the combination of electrophysiological, structural, and hemodynamic data, the influence of which is controlled by a set of hyperparameters, results in more accurate and reliable solutions than two commonly used source localization approaches. Future assessments of the IBF approach will require more realistic data. The use of simulated EEG data with added neurophysiologically realistic noise will necessitate the control of the solution for the noise level (hyperparameter $\lambda$ in Eq. 13 or 14). The value of the hyperparameter $\lambda$ can be estimated systematically by using an iterative restricted maximum likelihood procedure (Patterson and Thompson, 1971; Harville, 1974). The use of IBF to reduce the size of the problem should render the iterative process more tractable. Ultimately the solution proposed here will need to be evaluated with real data. These developments and applications are the subject of current research.

\section{REFERENCES}

Aine, C, Huang, M., Stephen, J., and Christner, R. 2000. Multistart algorithms for MEG empirical data analysis reliably characterize locations and time courses of multiple sources. Neurolmage 12: 159-172, doi:10.1006/nimg.2000.0616.

Ashburner, J., and Friston, K. J. 1997. Multimodal image coregis-tration and partitioning-A unified framework. Neurolmage 6: 209-217, doi:10.1006/nimg. 1997.0290.

Backus, G. E., and Gilbert, J. F. 1970. Uniqueness in the inversion of inacurate gross earth data. Phil. Trans. R. Soc. 266: 123-192.

Baillet, S., and Garnero, L. 1997. A Bayesian approach to introducing anatomo-functional priors in the EEG/MEG inverse problem. IEEE Trans. Biomed. Eng. 44: 374-385.

Baillet, S., Garnero, L., Marin, G., and Hugonin, J-P. 1999. Combined MEG and EEG source imaging by minimization of mutual information. IEEE Trans. Biomed. Eng. 46: 522-534.

Brooks, D. H., Ahmad, G. F., MacLeod, R. S., and Maratos, G. M. 1999. Inverse electrocardiography by simultaneous imposition of multiple constraints. IEEE Trans. Biomed. Eng. 46: 3-17.

Dale, A. M., and Sereno, M. I. 1993. Improved localisation of cortical activity by combining EEG and MEG with MRI cortical surface reconstruction: A linear approach. J. Cogn. Neurosci. 5: 162-176.

Dale, A. M., Fischl, B., and Sereno, M. I. 1999. Cortical surface-based analysis I: Segmentation and surface reconstruction. Neurolmage 9: 179-194, doi:10.1006/nimg.1998.0395.

Dale, A. M., Liu, A. K., Fischl, B. R., Buckner, R. L., Belliveau, J. W., Lewine, J. D., and Halgren, E. 2000. Dynamic statistical parametric mapping: Combining fMRI and MEG for high-resolution imaging of cortical activity. Neurone 26: 55-67.

Dempster, A. P., Laird, N. M., and Rubin, D. B. 1977. Maximum likelihood estimation from incomplete data via the EM algorithm. J. R. Stat. Soc. Ser. B 39: 1-38.

Ferguson, A. S., and Stroink, G. 1997. Factors affecting the accuracy of the boundary element method in the forward problem-I: Calculating surface potential. IEEE Trans. Biomed. Eng. 44: 1139-1155.

Fischl, B., and Dale, A. M. 2000. Mesuring the thickness of the human cerebral cortex from magnetic resonance images. Proc. Natl. Acad. Sci. USA 97: 11050-11055.

Fischl, B., Sereno, M. I., and Dale, A. M. 1999. Cortical surface-based analysis II: Inflation, flattening, and a surface-based coordinate system. Neurolmage 9: 195-207, doi:10.1006/nimg. 1998.0396.

Frackowiak, R. S. J., Friston, K. J., Frith, C. D., Dolan, R., and Mazziotta, J. C. 1997. Human Brain Function. Academic Press, San Diego. 
Fuchs, M., Wagner, M., Wischmann, HA., Köhler, T., Tiessen, A., Drenckhahn, R., and Buchner, H. 1998. Improving source reconstructions by combining bioelectric and biomagnetic data. Electro-encephalogr. Clin. Neurophysiol. 107: 93-111.

George, J. S., Aine, C. J., Mosher, J. C, Ranken, D. M., chlitt, H. A., Wood, C. C, Lewine, J. D., Sanders, J. A., and Belliveau., J. W. 1995. Mapping function in the human brain with MEG, anatomical MRI, and functional MRI. J. Clin. Neurophysiol. 12: 406-431.

Goebel, R., and Max Planck Society. 2000. Brain Voyager 2000, Version 4.2. http://www.brainvoyager.de.

Grave de Peralta Menendez, R., and Gonzalez Andino, S. 1998. A critical analysis of linear inverse solutions to the neuroelectromag-netic inverse problem. IEEE Trans. Biomed. Eng. 45: 440-448.

Grave de Peralta Menendez, R., and Gonzalez Andino, S. 1999. Backus and Gilbert method for vector fields. Human Brain Mapp. 7: 161-165.

Grave de Peralta Menendez, R., Hauk, O., Gonzalez Andino, S., Vogt, H., and Michel, C. 1997. Linear inverse solutions with optimal resolution kernels applied to electromagnetic tomography. Human Brain Mapp. 5: 454467.

Greenblatt, R. E. 1993. Probabilistic reconstruction of multiple sources in the bioelectromagnetic inverse problem. Inverse Problems 9: 271-284.

Hämäläinen, M. S., and Ilmoniemi, R. J. 1994. Interpreting magnetic fields of the brain: Minimum norm estimates. Med. Biol. Eng. Comput. 32: 35-42.

Hämäläinen, M. S., and Sarvas, J. 1989. Realistic conductivity geometry model of the human head for interpretation of neuromag-netic data. IEEE Trans. Biomed. Eng. 36: 165-171.

Hàmàlàinen, M. S., Hari, R., Ilmoniemi, R. J., Knuutila, J., and Lounasmaa, O. V. 1993.

Magentoencephalography_-Theory, instrumentation, and applications to noninvasive studies of the working human brain. Rev. Modern Phys. 65: 413-497.

Harville, D. A. 1974. Bayesian inference for variance components using only error contrasts. Biometrika 61: 383-385.

Huang, M., Aaron, R., and Shiffman, C. A. 1997. Maximum entropy method for magnetoencephalography. IEEE Trans. Biomed. Eng. 44: 98-102.

Jones, D. S. 1979. Elementary Information Theory. Clarendon, Oxford.

Kiebel, S. J., Goebel, R., and Friston, K. J. 2000. Anatomically informed basis functions. Neurolmage 11: 656667, doi:10.1006/ nimg.1999.0542.

Liu, A. K., Belliveau, J. W., and Dale, A. M. 1998. Spatiotemporal imaging of human brain activity using functional MRI constrained magentoencephalography data: Monte Carlo simulations. Proc. Nat. Acad. Sci. USA 95: 8945-8950.

Malmivuo, J., and Plonsey, R. 1995. Bioelectromagnetism: Principles and Applications of Bioelectric and Biomagnetic Fields. Oxford Univ. Press, London.

Miltner, W., Braun, C, Johnson, R., Simpson, G. V., and Ruchkni, D. S. 1994. A test of brain electrical source analysis (BESA): A simulation study. Electroencephalogr. Clin. Neurophysiol. 91: 295-310.

Mosher, J. C, Lewis, P. S., and Leahy, R. M. 1992. Multiple dipole modelling and localization from spatiotemporal MEG data. IEEE Trans. Biomed. Eng. 39: 541-557.

Nunez, P. L. 1981. Electric Fields of the Brain: The Neurophysics of EEC Oxford Univ. Press, New York.

Opitz, B., Mecklinger, A., Friederici, A. D., and von Cramon, D. Y. 1999. The functional neuroanatomy of novelty processing: Integrating ERP and fMRI results. Cerebral Cortexd: 379-391.

Pascual-Marqui, R. D. 1995. Reply to comments by M. Hàmàlàinen, R. Ilmoniemi and P. Nunez. In Source Localization: Continuing

Discussion of the Inverse Prolem (W. Skrandies, Ed.), pp. 16-28. ISBET Newsletter, No. 6.

Pascual-Marqui, R. D. 1999. Review of methods for solving the EEG inverse problem. Int. J. Bioelectromagn. 1: 75-86.

Pascual-Marqui, R. D., Michel, C. M., and Lehmann, D. 1994. Low resolution electromagnetic tomography: A 
new method for localizing electrical activity in the brain. Int. J. Psychophysiol. 18: 49-65.

Patterson, H. D., and Thompson, R. 1971. Recovery of inter-block information when block sizes are unequal. Biometrika 58: 545-554.

Perona, P., and Malik, J. 1990. Scale-space and edge detection using anisotropic diffusion. IEEE Trans. Pattern Anal. Machine Intelligence 12: 629-639.

Phillips, C, Rugg, M. D., and Friston, K. J. 2002. Systematic Noise Regularisation for Linear Inverse Solution of the Source Localisation Problem in EEG.

Rao, C. R., and Mitra, S. K. 1973. Theory and application of constrained inverse of matrices. SIAM J. Appl. Math. 24: 473-488.

Rugg, M. D. 1998. Convergent approaches to electrophysiological and haemodynamics investigations of memory. Human Brain Mapp. 6: 394-398.

Rugg, M. D. 1999. Functional neuroimaging in cognitive neuroscience. In The Neurocognition of Language (C. M. Brown and P. Hagoort, Eds.), pp. 15-36. Oxford Univ. Press, Oxford.

Sarvas, J. 1987. Basic mathematical and electromagnetic concepts of the biomagnetic inverse problem. Phys. Med. Biol. 32: 11-22.

Scherg, M., and Ebersole, J. S. 1994. Brain source imaging of focal and multifocal epileptiform EEG activity. Clin. Neurophysiol. 24: 51-60.

Scherg, M., Bast, T., and Berg, P. 1999. Multiple source analysis of interictal spikes: Goals, requirements, and clinical value. J. Clin. Neurophysiol. 16: 214-224.

Tikhonov, A. N., and Arsenin, V. Y. 1977. Solutions of I11-Posed Problems. Wiley, New York.

Uutela, K., Hämäläinen, M. S., and Somersalo, E. 1999. Visualization of magnetoencephalographic data using minimum current estimates. Neurolmage 10: 173-180, doi: 10.1006/nimg. 1999.0454.

von Helmholtz, H. L. F. 1853. Ueber einige Gesetze der Vertheilung elektrischer Ströme in köperlichen Leitern mit Anwendung auf die thierisch-elektrischen Versuche. Ann. Phys. Chem. 89: 211-233, 354-377.

Wellcome Department of Cognitive Neurology. 1999. Statistical Parametric Mapping, SPM'99. http://www.fil.ion.ucl.ac.uk/spm/ spm99.html. 